\title{
Non-supersymmetric, multi-center solutions with topological flux
}

\author{
Benjamin E. Niehoff \\ Department of Physics and Astronomy, University of Southern California, \\ 825 Bloom Walk, Los Angeles, CA 90089, U.S.A. \\ E-mail: bniehoff@usc.edu
}

ABSTRACT: We find an infinite class of non-supersymmetric multi-center solutions to the STU model in five-dimensional ungauged supergravity coupled to two vector multiplets. The solutions are obtained by solving a system of linear equations on a class of Ricci-scalarflat Kähler manifolds studied by LeBrun. After imposing an additional U(1) isometry in the base, we solve the axisymmetric $\mathrm{SU}(\infty)$ Toda equation and obtain explicit supergravity solutions containing arbitrary numbers of 2-cycles with cohomological fluxes of all three flavors. This improves upon a previous result where only two of the three fluxes were topologically non-trivial. Imposing regularity and absence of closed timelike curves, we obtain "bubble equations" highly reminiscent of those known in the supersymmetric case. Thus we extend much of the analysis done for BPS bubbling solutions to this new family of non-supersymmetric bubbling solutions.

KeYwords: Black Holes in String Theory, D-branes, M-Theory

ARXIV EPRINT: 1308.6335 


\section{Contents}

1 Introduction 1

2 Non-BPS solutions from floating branes 3

3 LeBrun metrics $\quad 5$

3.1 Topological structure 5

$\begin{array}{lll}3.2 & \text { As Euclidean-Einstein-Maxwell solutions } & 6\end{array}$

$\begin{array}{lll}3.3 & \text { Floating branes on a LeBrun base } & 7\end{array}$

4 Axisymmetric Kähler base spaces $\quad 8$

4.1 Solving the axisymmetric Toda equation 8

$\begin{array}{ll}4.2 \text { Boundary conditions } & 10\end{array}$

$\begin{array}{lll}4.3 & \text { Near the singularities } & 12\end{array}$

$\begin{array}{lll}\text { 4.3.1 Specific details of the groups } G & 15\end{array}$

$\begin{array}{lll}4.4 \text { At infinity } & 16\end{array}$

4.5 Ambipolar bases 16

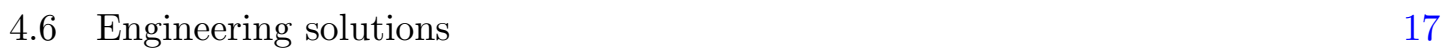

4.6.1 Example 1: every interior $\widetilde{q}_{i}=1$, but at infinity $\widetilde{q}_{\infty}=-1 \quad 18$

4.6.2 Example 2: every interior $\widetilde{q}_{i} \geq 1$, but at infinity $\widetilde{q}_{\infty}=+1 \quad 19$

$\begin{array}{lll}4.7 \text { A topological ménagerie } & 19\end{array}$

$\begin{array}{ll}4.8 \text { Magnetic flux through cycles } & 21\end{array}$

5 Multi-centered supergravity solutions $\quad 22$

5.1 Asymptotics of the 5d metric 22

5.1.1 Asymptotics for lifting to 11d SUGRA 23

5.1.2 Asymptotics lifting to IIB on $T^{4} \quad 24$

$\begin{array}{lll}5.2 & \text { Regularity conditions } & 25\end{array}$

$\begin{array}{ll}5.3 \text { Fluxes through cycles } & 26\end{array}$

5.4 Causality conditions: the "bubble equations" 26

$\begin{array}{ll}5.5 \text { An explicit numerical example } & 29\end{array}$

6 Conclusions $\quad 32$

A Groups at conical points from lattices in $\mathrm{SO}(4) \quad 34$

A.1 Orbifold points and more general conical singularities 34

A.1.1 Reduction to Smith normal form 35

A.2 The conical points of LeBrun metrics 37

A.2.1 When is a conical point an orbifold point? 38

A.2.2 When is the group $G$ trivial? 38

A.2.3 When is the group $G$ like a Gibbons-Hawking orbifold group? 38 


\section{Introduction}

In the past few years, there have been many exciting developments in the program of finding black hole microstate geometries. These are solitonic solutions to supergravity theories which have the same asymptotic behavior as a given black hole (or black ring), including mass, charge, and angular momentum, and yet in the bulk remain totally smooth and free of horizons. Instead, the pathological parts of the would-be black hole are resolved by a collection of smooth, topological bubbles, threaded by cohomological fluxes which hold the whole thing up against gravitational collapse. It is conjectured that such geometries may provide the "hair" necessary to store the entropy of the black hole (or black ring) $[1,2]$, and can be interpreted as supergravity approximations to the stringy states (or "fuzzballs") thought to resolve the information paradox [3]. Beyond specifically finding smooth microstate geometries, this program is of general interest for providing numerous examples of stationary supergravity solutions containing arbitrary collections of charged, rotating black holes and rings balanced by their mutual electromagnetic interactions.

Of central importance to this program is the discovery that the BPS equations for 5-dimensional, $\mathcal{N}=2$ supergravity coupled to vector multiplets can be cast as a linear system [4]. From this came a whole body of work on BPS solutions, extending previouslyknown families of solutions and uncovering new ones; especially leading to the construction of the "bubbling microstate geometries", or solitons made of pure topological bubbles and fluxes [5-8]. These solutions are constructed with a time fiber over a hyper-Kähler Gibbons-Hawking (GH) base [9], which contains topologically non-trivial 2-cycles supported by harmonic fluxes. In particular, one finds a set of "bubble equations", which arise from demanding the absence of closed timelike curves. The bubble equations relate the cohomological fluxes to the sizes of the homological bubbles to which they are linked; thus, the bubbles are literally held open by the fluxes.

More recently, there have been several attempts to get away from BPS. A few isolated examples exist [10-13] of truly non-BPS, non-extremal smooth geometries, but no infinite familes are yet known (which are necessary for entropy counting). However, in the nonBPS extremal case, there are linear systems which can be solved to obtain infinite families of solutions. One such family are the so-called "almost BPS" solutions [14-16], where supersymmetry is broken by inverting the orientation of the Gibbons-Hawking base relative to the fluxes. These solutions have been shown to exhibit a rich variety of phenomena not seen in the BPS case [17-19].

A further avenue of attack was revealed with the "floating brane" ansatz in 5 dimensions, which dispenses with supersymmetry, but still imposes a generic balance between gravitational and electromagnetic forces. It was found that this leads to yet another linear system of equations, this time on a Euclidean-signature Einstein-Maxwell base [20]. A few 
solutions are known based on various Euclidean-Einstein-Maxwell geometries analytically continued from classical GR ones [21], as well as an infinite family given in [20] based on the Israel-Wilson metric.

In a pair of recent papers $[22,23]$, the author and collaborators have presented an infinite family of "floating brane" non-BPS solutions based on a family of Kähler EinsteinMaxwell metrics studied by LeBrun [24, 25]. These metrics are determined by two functions which solve the $\mathrm{SU}(\infty)$ Toda equation and its linearization. By choosing an extremely simple solution to the Toda equation, one obtains the subclass of LeBrun-Burns metrics, which are Kähler analogues to Gibbons-Hawking metrics with a hyperbolic base instead of flat $\mathbb{R}^{3}$. On the LeBrun-Burns base, the floating brane equations are solvable and one obtains an infinite family of solutions.

These solutions were shown to have a few desirable properties. The LeBrun-Burns metrics have the structure of a $\mathrm{U}(1)$ fiber over $\mathbb{H}^{3}$. In much the same way as GibbonsHawking metrics, this U(1) fiber pinches off at controlled points, which allows one to construct solutions with several "bubbles" threaded with cohomological fluxes. It was also shown that with appropriate choices of parameters, the solutions could be made regular and free of CTC's.

However, these solutions also had a few shortcomings. The Maxwell field of the LeBrunBurns metrics is topologically trivial. Hence, while one can use the U(1) fiber to form 2cycles, only two of the three fluxes thread those 2-cycles. The resulting "bubble equations" turn out to be independent of the sizes of the bubbles, and thus the interplay between bubbles and fluxes is gone. Furthermore, the solution is very degenerate, because it effectively has only two types of dipole charges. As a result, the regularity conditions actually demand that most of the parameters be set to zero. Finally, the solutions are not asymptotically flat; in fact, it was shown that the floating brane equations on a Kähler base have no asymptotically-flat solutions in general [22]. This last shortcoming should not be all too great a concern. One does obtain solutions whose asymptotics are like the near-horizon limit of a BMPV black hole [26]. So it is not too far a stretch to say that these are BMPV microstate geometries, and probably the asymptotic region can be restored by relaxing the assumptions of the floating brane ansatz.

Yet another linear system of equations was discovered by re-organizing the BPS equations in the 6-dimensional IIB frame [27-29], which makes a curious connection to the 5-dimensional story: the 5-dimensional non-BPS, floating brane equations on a Kähler base are identical to the 6-dimensional BPS equations where all functions are made independent of the 6th coordinate [23]. Therefore the exact same family of solutions plays two roles, both supersymmetric and non-supersymmetric. The apparent discrepancy is explained in the KK reduction from 6 to 5 dimensions: the Killing spinor in 6 dimensions can be charged under the $\mathrm{U}(1)$ on which the reduction occurs, which causes it to vanish in 5 dimensions. This is reminiscent of the Scherk-Schwarz mechanism [30,31], or also "supersymmetry without supersymmetry" [32].

In this paper, we improve upon the results of [22] and overcome its major issues. Despite the 5d-6d link mentioned, we work strictly in the 5-dimensional frame, as it is the simpler of the two. This paper is organized as follows: in section 2, we briefly describe the 
$5 \mathrm{~d} \mathcal{N}=2$ theory, the floating brane ansatz, and the equations that result. In section 3 , we describe the basic features of LeBrun metrics in general, and the system that results from putting the floating brane equations on the LeBrun base. We show how the system is solved generically. In section 4 , we solve the $\mathrm{SU}(\infty)$ Toda equation explicitly under the assumption of an additional U(1) isometry. We determine the boundary conditions needed for the solutions we wish to build, and we analyze the resulting base manifold in detail to explore its geometric and topological properties. In section 5, we solve the floating brane equations on this base manifold explicitly, thus giving the full supergravity solution. We determine the conditions needed to make solutions regular in 5 dimensions. We derive the no-CTC conditions, or "bubble equations" and analyze them. Finally, we give an explicit, solved example of a 3 -center solution. In section 6 , we present our conclusions.

\section{Non-BPS solutions from floating branes}

It is simplest to present our solutions in the context of $\mathcal{N}=2$ ungauged supergravity in 5 dimensions coupled to two vector multiplets (thus having three $\mathrm{U}(1)$ gauge fields). One can also see this theory as a truncation of eleven-dimensional supergravity on $T^{6}$. The 5 -dimensional action is

$$
S=\frac{1}{2 \kappa_{5}} \int\left(\underset{5}{\star} \mathcal{R}-Q_{I J} \mathrm{~d} X^{I} \wedge \underset{5}{\star} \mathrm{d} X^{J}-Q_{I J} F^{I} \wedge \underset{5}{\star} F^{J}-\frac{1}{6} C_{I J K} F^{I} \wedge F^{J} \wedge A^{K}\right),
$$

where $\mathcal{R}$ is the Ricci scalar, $X^{I}, I \in\{1,2,3\}$ are scalar fields, $F^{I} \equiv d A^{I}$ are three Maxwell fields, and the kinetic terms are coupled via the matrix

$$
Q_{I J} \equiv \frac{1}{2} \operatorname{diag}\left(\left(X^{1}\right)^{-2},\left(X^{2}\right)^{-2},\left(X^{3}\right)^{-2}\right) .
$$

The scalar fields are subject to the constraint $X^{1} X^{2} X^{3}=1$, which we parametrize in terms of a new set of scalars $Z_{I}$ as

$$
X^{1}=\left(\frac{Z_{2} Z_{3}}{Z_{1}^{2}}\right)^{1 / 3}, \quad X^{2}=\left(\frac{Z_{1} Z_{3}}{Z_{2}^{2}}\right)^{1 / 3}, \quad X^{3}=\left(\frac{Z_{1} Z_{2}}{Z_{3}^{2}}\right)^{1 / 3}
$$

These new scalars $Z_{I}$ are very convenient in the ansätze to follow.

We begin with the usual $5 \mathrm{~d}$ metric ansatz,

$$
\mathrm{d} s_{5}^{2}=-Z^{-2}(\mathrm{~d} t+k)^{2}+Z \mathrm{~d} s_{4}^{2}, \quad Z \equiv\left(Z_{1} Z_{2} Z_{3}\right)^{1 / 3}
$$

with $4 \mathrm{~d}$ base manifold $\mathrm{d} s_{4}^{2}$. Following [20], the Maxwell fields are then given by the "floating brane" ansatz,

$$
A^{I} \equiv-Z_{I}^{-1}(\mathrm{~d} t+k)+B^{I}
$$

and it is convenient to introduce the magnetic 2 -forms given by

$$
\Theta^{(I)} \equiv \mathrm{d} B^{I}
$$


For completeness, we also give the embedding into 11-dimensional supergravity. The 11dimensional metric and 3-form potential are given by

$$
\begin{gathered}
\mathrm{d} s_{11}^{2}=\mathrm{d} s_{5}^{2}+\left(\frac{Z_{2} Z_{3}}{Z_{1}^{2}}\right)^{1 / 3}\left(\mathrm{~d} y_{1}^{2}+\mathrm{d} y_{2}^{2}\right)+\left(\frac{Z_{1} Z_{3}}{Z_{2}^{2}}\right)^{1 / 3}\left(\mathrm{~d} y_{3}^{2}+\mathrm{d} y_{4}^{2}\right) \\
+\left(\frac{Z_{1} Z_{2}}{Z_{3}^{2}}\right)^{1 / 3}\left(\mathrm{~d} y_{5}^{2}+\mathrm{d} y_{6}^{2}\right), \\
C^{(3)}=A^{1} \wedge \mathrm{d} y_{1} \wedge \mathrm{d} y_{2}+A^{2} \wedge \mathrm{d} y_{3} \wedge \mathrm{d} y_{4}+A^{3} \wedge \mathrm{d} y_{5} \wedge \mathrm{d} y_{6},
\end{gathered}
$$

where we see that the three scalars $X^{I}(2.3)$ come from the sizes of three $T^{2}$ 's inside the $T^{6}$ spanned by the coordinates $y_{i}$. In particular, for the $T^{6}$ to remain compact, the $Z_{I}$ must be everywhere finite and nonzero; or, if any of the $Z_{I} \rightarrow 0$ or $Z_{I} \rightarrow \infty$, they must all do so with the same behavior.

Returning to the 5-dimensional theory, as was shown in [20], we then need a 4dimensional base manifold that solves the Euclidean-signature Einstein-Maxwell equations,

$$
R_{\mu \nu}^{(4)}=\frac{1}{2}\left(\mathcal{F}_{\mu \rho} \mathcal{F}_{\nu}{ }^{\rho}-\frac{1}{4} g_{\mu \nu} \mathcal{F}_{\rho \sigma} \mathcal{F}^{\rho \sigma}\right)
$$

where $\mathcal{F}$ is a Maxwell 2-form determined by the base geometry, and unrelated to the $F^{I}$. We decompose $\mathcal{F}$ as

$$
\mathcal{F} \equiv \Theta^{(3)}-\omega_{-}^{(3)},
$$

where $\Theta^{(3)}$ is self-dual, and $\omega_{-}^{(3)}$ is anti-self-dual. The Maxwell equations $\mathrm{d} \mathcal{F}=\mathrm{d} \star_{4} \mathcal{F}=0$ imply that $\Theta^{(3)}$ and $\omega_{-}^{(3)}$ are harmonic. As the notation implies, this defines the magnetic 2-form field strength $\Theta^{(3)}$.

The equations of motion of (2.1) then reduce to the linear system [20]:

$$
\begin{array}{ll}
\mathrm{d}_{4}^{\star \mathrm{d}} Z_{1}=\Theta^{(2)} \wedge \Theta^{(3)}, & \Theta^{(2)}-\underset{4}{\star} \Theta^{(2)}=2 Z_{1} \omega_{-}^{(3)}, \\
\mathrm{d}_{4}^{\star \mathrm{d}} Z_{2}=\Theta^{(1)} \wedge \Theta^{(3)}, & \Theta^{(1)}-\underset{4}{\star} \Theta^{(1)}=2 Z_{2} \omega_{-}^{(3)},
\end{array}
$$

and

$$
\begin{aligned}
\mathrm{d}_{4}^{\star \mathrm{d}} Z_{3} & =\Theta^{(1)} \wedge \Theta^{(2)}-\omega_{-}^{(3)} \wedge(\mathrm{d} k-\underset{4}{\star \mathrm{d} k} k), \\
\mathrm{d} k+\underset{4}{\star} \mathrm{d} k & =\frac{1}{2} \sum_{I} Z_{I}\left(\Theta^{(I)}+\underset{4}{\star} \Theta^{(I)}\right) .
\end{aligned}
$$

We solve the equations of motion by the following steps: first, find a Euclidean-EinsteinMaxwell base. The Maxwell 2-form defines the 2-forms $\Theta^{(3)}$ and $\omega_{-}^{(3)}$ via (2.10). We then solve the first layer of coupled linear equations (2.11) and (2.12) for $Z_{1}, Z_{2}, \Theta^{(1)}$, and $\Theta^{(2)}$. These enter as sources in the second layer of coupled linear equations (2.13) and (2.14), which we solve finally for $Z_{3}$ and $k$. Next we follow [22, 23] and implement this solution for the LeBrun metrics. 


\section{LeBrun metrics}

The LeBrun family of metrics [24] is given by

$$
g \equiv \frac{1}{w}(\mathrm{~d} \tau+A)^{2}+w e^{u}\left(\mathrm{~d} x^{2}+\mathrm{d} y^{2}\right)+w \mathrm{~d} z^{2},
$$

where $\tau$ is periodic with period $4 \pi$. The functions $u$ and $w$ are independent of $\tau$ and solve the $\mathrm{SU}(\infty)$ Toda equation and its linearization, respectively:

$$
\begin{aligned}
u_{x x}+u_{y y}+\left(e^{u}\right)_{z z} & =0, \\
w_{x x}+w_{y y}+\left(e^{u} w\right)_{z z} & =0,
\end{aligned}
$$

and the 1-form $A$ satisfies

$$
\mathrm{d} A=w_{x} \mathrm{~d} y \wedge \mathrm{d} z+w_{y} \mathrm{~d} z \wedge \mathrm{d} x+\left(e^{u} w\right)_{z} \mathrm{~d} x \wedge \mathrm{d} y
$$

Under the conditions (3.3) and (3.4), the metric (3.1) is Kähler, with Kähler form

$$
J \equiv(\mathrm{d} \tau+A) \wedge \mathrm{d} z-e^{u} w \mathrm{~d} x \wedge \mathrm{d} y
$$

The condition (3.2) further implies that the Ricci scalar vanishes [24].

We choose to introduce the frames,

$$
e^{1}=w^{-1 / 2}(\mathrm{~d} \tau+A), \quad e^{2}=e^{u / 2} w^{1 / 2} \mathrm{~d} x, \quad e^{3}=e^{u / 2} w^{1 / 2} \mathrm{~d} y, \quad e^{4}=w^{1 / 2} \mathrm{~d} z
$$

with orientation

$$
\operatorname{vol}_{4} \equiv e^{1} \wedge e^{2} \wedge e^{3} \wedge e^{4}=e^{u} w \mathrm{~d} \tau \wedge \mathrm{d} x \wedge \mathrm{d} y \wedge \mathrm{d} z
$$

such that $J$ is anti-self-dual. It will also be helpful to define the (anti)-self-dual 2-forms

$$
\begin{array}{ll}
\Omega_{ \pm}^{(1)}=e^{-u / 2}\left(e^{1} \wedge e^{2} \pm e^{3} \wedge e^{4}\right) & =(\mathrm{d} \tau+A) \wedge \mathrm{d} x \pm w \mathrm{~d} y \wedge \mathrm{d} z \\
\Omega_{ \pm}^{(2)}=e^{-u / 2}\left(e^{1} \wedge e^{3} \pm e^{4} \wedge e^{1}\right) & =(\mathrm{d} \tau+A) \wedge \mathrm{d} y \pm w \mathrm{~d} z \wedge \mathrm{d} x \\
\Omega_{ \pm}^{(3)}=e^{1} \wedge e^{4} \pm e^{2} \wedge e^{3} & =(\mathrm{d} \tau+A) \wedge \mathrm{d} z \pm w e^{u} \mathrm{~d} x \wedge \mathrm{d} y
\end{array}
$$

such that $J=\Omega_{-}^{(3)}$.

\subsection{Topological structure}

The LeBrun metrics (3.1) have the structure of a U(1) fiber over a 3-dimensional base with metric

$$
h=e^{u}\left(\mathrm{~d} x^{2}+\mathrm{d} y^{2}\right)+\mathrm{d} z^{2},
$$

which in turn can be thought of as a Riemann surface fibered over a line. If $e^{u}$ is everywhere finite and non-singular, then the $(x, y, z)$ coordinates can be extended to a topological $\mathbb{R}^{3}$. In this case, the topology of the 4-manifold can be analyzed in terms of the U(1) fiber parametrized by $\tau$, much like the topology of Gibbons-Hawking manifolds [9]. 


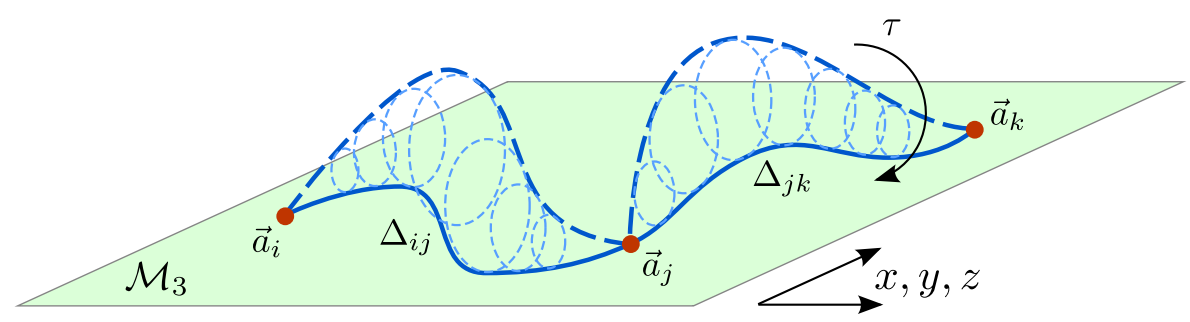

Figure 1. Homological 2-cycles in the LeBrun metric. The $\tau$ fiber pinches off at the points $\vec{a}_{i}$. Sweeping the fiber along a path between any two points forms a homological 2-sphere. Two intersecting 2-cycles are shown.

The function $w$ solves a second-order Laplace-like equation, whose solutions are characterized by a number of points we will call "Gibbons-Hawking points" or "geometric charges", where locally (provided that $e^{u}$ is smooth),

$$
w \sim \frac{1}{r}
$$

for some local radial distance $r$. At these points the $\tau$ fiber pinches off to zero size. Hence, if one takes any curve in the 3-dimensional base $h$ that joins two geometric charges, the surface described by the $\tau$ fiber over this curve is a homological 2-sphere, as in figure 1 .

If $e^{u}$ is not smooth, it is still possible that $g$ is smooth. One possibility is that $z$ is a radial coordinate, and $e^{u}\left(\mathrm{~d} x^{2}+\mathrm{d} y^{2}\right)$ describes a sphere (or perhaps a quotient of a sphere). Another possibility is that $e^{u}\left(\mathrm{~d} x^{2}+\mathrm{d} y^{2}\right)$ is a higher-genus Riemann surface, in which case one can have topological cycles that do not involve the $\tau$ fiber. Some of these additional topological features will appear in the solutions presented in this paper.

\subsection{As Euclidean-Einstein-Maxwell solutions}

One can show $[22,23]$ that self-dual, harmonic 2-forms on LeBrun spaces can be written

$$
\Theta \equiv \sum_{a=1}^{3} \partial_{a}\left(\frac{H}{w}\right) \Omega_{+}^{(a)}=(\mathrm{d} \tau+A) \wedge \mathrm{d} \frac{H}{w}+w \underset{3}{w} \frac{H}{w}
$$

where $H$ solves $(3.3)$ and $\star_{3}$ is taken with respect to the 3 -metric

$$
h=e^{u}\left(\mathrm{~d} x^{2}+\mathrm{d} y^{2}\right)+\mathrm{d} z^{2} .
$$

By differentiating (3.2) with respect to $z$, one can show that $u_{z}$ solves (3.3). So define the Maxwell 2-form

$$
\mathcal{F} \equiv \Theta+\alpha J, \quad \text { with } \quad H=-\frac{u_{z}}{2 \alpha} .
$$

Then $(g, \mathcal{F})$ solve the Euclidean-Einstein-Maxwell equations [25].

For simplicity in matching with the linear system found in [20], we choose $\alpha=-1$, and hence

$$
\Theta^{(3)}=\frac{1}{2}(\mathrm{~d} \tau+A) \wedge \mathrm{d} \frac{u_{z}}{w}+\frac{1}{2} w \underset{3}{ } \mathrm{~d}_{\frac{u_{z}}{w}}, \quad \omega_{-}^{(3)}=J .
$$




\subsection{Floating branes on a LeBrun base}

Next we solve the system (2.11), (2.12), (2.13), (2.14) on the LeBrun base. We will find it convenient to define

$$
K^{3} \equiv \frac{u_{z}}{2}, \quad \text { such that } \quad \Theta^{(3)}=(\mathrm{d} \tau+A) \wedge \mathrm{d} \frac{K^{3}}{w}+w \underset{3}{*} \mathrm{~d} \frac{K^{3}}{w} .
$$

To solve the first layer, one makes the ansätze

$$
\begin{array}{ll}
\Theta^{(1)}=(\mathrm{d} \tau+A) \wedge \mathrm{d} \frac{K^{1}}{w}+w \star \mathrm{d}_{3} \mathrm{~d} \frac{K^{1}}{w}+Z_{2}\left(\Omega_{-}^{(3)}-\Omega_{+}^{(3)}\right), & Z_{2}=\frac{K^{1} K^{3}}{w}+L_{2}, \\
\Theta^{(2)}=(\mathrm{d} \tau+A) \wedge \mathrm{d} \frac{K^{2}}{w}+w \star \mathrm{d}_{3} \frac{K^{2}}{w}+Z_{1}\left(\Omega_{-}^{(3)}-\Omega_{+}^{(3)}\right), & Z_{1}=\frac{K^{2} K^{3}}{w}+L_{1} .
\end{array}
$$

This leads to the linear equations

$$
\partial_{x}^{2} L_{1}+\partial_{y}^{2} L_{1}+\partial_{z}^{2}\left(e^{u} L_{1}\right)=0, \quad \partial_{x}^{2} L_{2}+\partial_{y}^{2} L_{2}+\partial_{z}^{2}\left(e^{u} L_{2}\right)=0,
$$

and

$$
\begin{aligned}
& \partial_{x}^{2} K^{1}+\partial_{y}^{2} K^{1}+\partial_{z}\left(e^{u} \partial_{z} K^{1}\right)=2 \partial_{z}\left(e^{u} w L_{2}\right), \\
& \partial_{x}^{2} K^{2}+\partial_{y}^{2} K^{2}+\partial_{z}\left(e^{u} \partial_{z} K^{2}\right)=2 \partial_{z}\left(e^{u} w L_{1}\right) .
\end{aligned}
$$

To solve the second layer, make the ansätze

$$
k=\mu(\mathrm{d} \tau+A)+\omega, \quad Z_{3}=\frac{K^{1} K^{2}}{w}+L_{3}, \quad \mu=-\frac{K^{1} K^{2} K^{3}}{w^{2}}-\frac{1}{2} \sum_{I=1}^{3} \frac{K^{I} L_{I}}{w}+M
$$

Then the new functions $M$ and $L_{3}$ satisfy the equations

$$
\begin{aligned}
\partial_{x}^{2} M+\partial_{y}^{2} M+\partial_{z}\left(e^{u} \partial_{z} M\right) & =\partial_{z}\left(e^{u} L_{1} L_{2}\right), \\
\partial_{x}^{2} L_{3}+\partial_{y}^{2} L_{3}+e^{u} \partial_{z}^{2} L_{3} & =4 e^{u} w L_{1} L_{2}-4 e^{u} w \partial_{z} M-2 e^{u}\left(L_{1} \partial_{z} K^{1}+L_{2} \partial_{z} K^{2}\right),
\end{aligned}
$$

and the 1-form $\omega$ satisfies

$$
\begin{aligned}
\mathrm{d} \omega= & w \underset{3}{\star} \mathrm{d} M-M_{3}^{\star} \mathrm{d} w-u_{z} w M_{3}^{\star} \mathrm{d} z-2 w L_{1} L_{2}{ }_{3}^{\star} \mathrm{d} z \\
& +\frac{1}{2} \sum_{I}\left(L_{I} \underset{3}{\star} \mathrm{d} K^{I}-K_{3}^{I} L_{I}\right)-\frac{1}{2} u_{z}\left(K^{1} L_{1}+K^{2} L_{2}\right) \underset{3}{\star \mathrm{d}} z+\frac{1}{2} u_{z} K^{3} L_{3} \star \mathrm{d}_{3} \mathrm{~d} z
\end{aligned}
$$

Therefore, to solve the "floating brane" system on the LeBrun base, one first finds a function $u$ that solves the $\mathrm{SU}(\infty)$ Toda equation, which also defines the function $K^{3} \equiv \frac{1}{2} u_{z}$. Then one solves (3.3), (3.20)-(3.22), (3.24) and (3.25), in this order, for the seven remaining functions $w, K_{1}, K_{2}, L_{1}, L_{2}, L_{3}$, and $M$. Finally, one must solve (3.26) for the 1-form $\omega$. 


\section{Axisymmetric Kähler base spaces}

Before we discuss solutions to the full system, we will explore the base space $g$ in detail. Our task is to solve the $\mathrm{SU}(\infty)$ Toda equation which, while known to be integrable, is also notoriously hard. However, if we impose an additional U(1) symmetry, there is a known method of attack [33-37].

First let us write the LeBrun metric in an explicitly $\mathrm{U}(1) \times \mathrm{U}(1)$-invariant form,

$$
g=\frac{1}{w}(\mathrm{~d} \tau+A)^{2}+w e^{u}\left(\mathrm{~d} r^{2}+r^{2} \mathrm{~d} \phi^{2}\right)+w \mathrm{~d} z^{2},
$$

where now all functions depend on $r, z$ only. For completeness, the equations to be solved in these coordinates become

$$
\begin{aligned}
\frac{1}{r} \partial_{r}\left(r u_{r}\right)+\left(e^{u}\right)_{z z} & =0, \\
\frac{1}{r} \partial_{r}\left(r w_{r}\right)+\left(e^{u} w\right)_{z z} & =0
\end{aligned}
$$

and

$$
\mathrm{d} A=r w_{r} \mathrm{~d} \phi \wedge \mathrm{d} z+\left(e^{u} w\right)_{z} r \mathrm{~d} r \wedge \mathrm{d} \phi .
$$

At this point, we can solve (4.3) and (4.4) generically. To accomplish this, note that the Laplacian on the 3-dimensional base $h$ is given by

$$
e^{u} \Delta_{h}(\varphi)=\frac{1}{r} \partial_{r}\left(r \varphi_{r}\right)+\left(e^{u} \varphi_{z}\right)_{z},
$$

and hence the Laplacian is related to the linearized Toda equation via $\partial_{z}$ :

$$
\partial_{z}\left(e^{u} \Delta_{h}(\varphi)\right)=\frac{1}{r} \partial_{r}\left(r \partial_{r} \varphi_{z}\right)+\left(e^{u} \varphi_{z}\right)_{z z} .
$$

Therefore if we take some $\hat{w}$ which solves the Laplace equation on $h$

$$
\frac{1}{r} \partial_{r}\left(r \hat{w}_{r}\right)+\left(e^{u} \hat{w}_{z}\right)_{z}=0,
$$

then it is easy to show that (4.3) and (4.4) are solved by

$$
w=\hat{w}_{z}, \quad A=-r \hat{w}_{r} \mathrm{~d} \phi .
$$

One can think of $\hat{w}$ as a "potential" that gives us the solutions for $w$ and $A$.

\subsection{Solving the axisymmetric Toda equation}

Now let us focus on the Toda equation with an axial symmetry (4.2). The additional U(1) symmetry allows one to make a Bäcklund transformation to new coordinates $\rho, \eta[33-37]$ :

$$
r^{2} e^{u}=\rho^{2}, \quad \log r=V_{\eta}, \quad z=-\rho V_{\rho} .
$$


The Toda equation can then be mapped ${ }^{1}$ onto the axisymmetric Laplace equation ${ }^{2}$ in $\mathbb{R}^{3}$ in cylindrical coordinates:

$$
\frac{1}{\rho} \partial_{\rho}\left(\rho V_{\rho}\right)+V_{\eta \eta}=0
$$

In principle, one must then invert the transformation (4.9) to obtain $u$. But in practice, for most functions $V$ this is intractable. It is easier to change the metric to the new coordinates $\rho, \eta$, which results in

$$
\begin{aligned}
g & =\frac{1}{w}(\mathrm{~d} \tau+A)^{2}+w h, \\
h & =\rho^{2}\left(V_{\rho \eta}^{2}+V_{\eta \eta}^{2}\right)\left(\mathrm{d} \rho^{2}+\mathrm{d} \eta^{2}\right)+\rho^{2} \mathrm{~d} \phi^{2} .
\end{aligned}
$$

We should note that as a result of the transformations (4.9), the cylindrical coordinates $\rho, \eta, \phi$ inherit the orientation opposite to the usual:

$$
\operatorname{vol}_{h}=\rho^{2}\left(V_{\rho \eta}^{2}+V_{\eta \eta}^{2}\right) \mathrm{d} \rho \wedge \mathrm{d} \eta \wedge \mathrm{d} \phi
$$

We must also change (4.3) and (4.4) into the new coordinates. The Laplacian $\Delta_{h}$ becomes, up to an overall factor, the cylindrically-symmetric Laplacian on $\mathbb{R}^{3}$,

$$
\rho^{2}\left(V_{\rho \eta}^{2}+V_{\eta \eta}^{2}\right) \Delta_{h}(\varphi)=\frac{1}{\rho} \partial_{\rho}\left(\rho \varphi_{\rho}\right)+\varphi_{\eta \eta}
$$

and so the potential $\hat{w}$ solves

$$
\frac{1}{\rho} \partial_{\rho}\left(\rho \hat{w}_{\rho}\right)+\hat{w}_{\eta \eta}=0
$$

whose solutions we know well. Then $w$ and $A$ are given by

$$
w=\hat{w}_{z}=\frac{1}{\rho\left(V_{\rho \eta}^{2}+V_{\eta \eta}^{2}\right)}\left(V_{\eta \eta} \hat{w}_{\rho}-V_{\rho \eta} \hat{w}_{\eta}\right) .
$$

and

$$
A=-r \hat{w}_{r} \mathrm{~d} \phi=-\frac{1}{V_{\rho \eta}^{2}+V_{\eta \eta}^{2}}\left(V_{\rho \eta} \hat{w}_{\rho}+V_{\eta \eta} \hat{w}_{\eta}\right) \mathrm{d} \phi .
$$

Therefore, the geometric data of the base space are determined in terms of two functions $V, \hat{w}$ that solve the axisymmetric Laplace equation in $\mathbb{R}^{3}$.

\footnotetext{
${ }^{1}$ We note that the mapping (4.9) from Toda onto Laplace is not one-to-one. The Toda equation maps onto a nonlinear equation in $V$ containing up to 3rd-order derivatives. That $V$ solves the Laplace equation is sufficient, but not necessary, for the solution of this nonlinear equation. Hence the family of Laplace solutions does not capture all possible Toda solutions under the inverse Bäcklund transformation.

${ }^{2}$ Strictly speaking, this is a Poisson equation and we have ignored subtleties involving source terms (supported on a locus of measure zero) on the right-hand side of (4.2). We avoid these subtleties by transforming the whole metric (taken as a local expression on an open chart) to the new coordinates $(\rho, \eta)$, while forgetting the old coordinates. In section 4.2 we will discuss the source terms in the new coordinates which should appear in the right-hand-side of (4.10). We remain agnostic about the exact form of the source terms as they would appear in the original coordinates (4.2), as this information is not necessary for constructing supergravity solutions.
} 


\subsection{Boundary conditions}

The task of writing an explicit base space is then reduced to solving cylindrically symmetric electrostatics problems in $\mathbb{R}^{3}[33]$. The question is what kinds of electrostatic problems give interesting solutions.

By analogy with BPS solutions on Gibbons-Hawking bases [7], we expect to specify a collection of points along the $\eta$ axis where $w$ and $K^{3} \equiv \frac{1}{2} u_{z}$ have poles. The poles of $w$ control where the $\tau$ fiber pinches off, thus creating a series of homology 2-cycles (provided that the 3 -dimensional base $h$ remain smooth at these points). The poles of $u_{z}$ control sources of $\Theta^{(3)}$. If $u_{z}$ has a pole where $w$ does not, we expect a singularity in the metric. But if $u_{z}$ has poles coincident with poles of $w$, we expect that the base geometry be smooth (up to orbifold identifications), and such poles should control the fluxes of $\Theta^{(3)}$ on the adjacent 2-cycles.

In the simplest case, we consider where $w$ and $u_{z}$ each have a single, coincident pole. Since both $w$ and $u_{z}$ solve the same elliptic linear PDE (3.3) (with the same boundary condition at infinity) and have only one "source point", it follows that $w$ and $u_{z}$ are proportional. Hence $\Theta^{(3)}=0$ and the metric is Ricci-flat (and therefore hyper-Kähler) - thus the metric (4.1) should be a Gibbons-Hawking metric, in alternative coordinates. ${ }^{3}$ Looking at (4.1), we identify $z$ as the radial coordinate from the source point, and take $r, \phi$ to be stereographic coordinates on an $S^{2}$. Hence we can write

$$
e^{u}=\frac{4 z^{2}}{\left(1+r^{2}\right)^{2}}, \quad u_{z}=\frac{2}{z}, \quad w=\frac{q}{z}
$$

where $q$ is any integer. Then as $z \rightarrow 0$, the metric (4.1) is simply the flat metric on $\mathbb{R}^{4} / \mathbb{Z}_{q}$. This gives the canonical example of coincident poles in $w, u_{z}$. We expect that near any location where $w, u_{z}$ both blow up, the metric will locally have this form.

To get a function $u_{z}$ with many poles, we should choose a cylindrically-symmetric Laplace solution $V$ that gives rise to the behavior in (4.18), and then use linearity to combine several solutions at centered at different points. Using the Bäcklund transformation (4.9), we have

$$
u_{z}=\frac{2 V_{\eta \eta}}{\rho^{2}\left(V_{\rho \eta}^{2}+V_{\eta \eta}^{2}\right)}=-\frac{2}{\rho V_{\rho}} \quad=\frac{2}{z},
$$

where the center equality is the boundary condition we need to satisfy near the source point in order for $u_{z}$ to have the appropriate singular behavior. We see that while the cylindrically-symmetric Laplace equation for $V(4.10)$ is linear, the boundary condition for $V$ is nonlinear. To solve this boundary condition, one can guess a few known possibilities for $V$. It turns out the appropriate choice is also the most obvious one to give a pole in the numerator:

$$
V_{\eta \eta}=\frac{1}{\sqrt{\rho^{2}+\eta^{2}}}
$$

\footnotetext{
${ }^{3}$ In the general LeBrun ansatz, taking $w \sim u_{z}$ gives not a Gibbons-Hawking metric, but a more general hyper-Kähler manifold. However, if we set $w \sim u_{z}$ in the $\mathrm{U}(1) \times \mathrm{U}(1)$-invariant ansatz of (4.1), there is always some linear combination of the U(1)'s which is tri-holomorphic, hence the manifold must in fact be Gibbons-Hawking but written in unusual coordinates.
} 


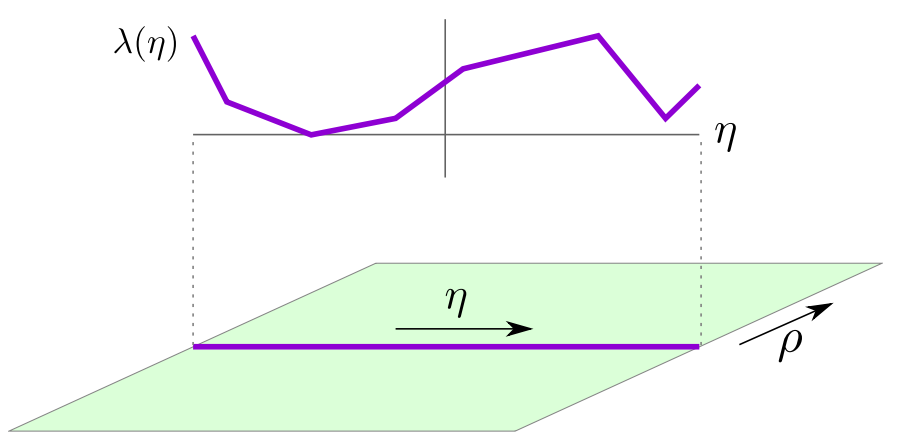

Figure 2. The electrostatics problem corresponding to $V . \lambda(\eta)$ is a line charge density profile along the $\eta$ axis, which is piecewise linear with "kinks" at each of the $\eta_{i}$.

Integrating this twice with respect to $\eta$ and choosing appropriate integration constants, we find

$$
V=-\sqrt{\rho^{2}+\eta^{2}}+\eta \log \frac{\eta+\sqrt{\rho^{2}+\eta^{2}}}{\rho} .
$$

Then we have

$$
z=-\rho V_{\rho}=\sqrt{\rho^{2}+\eta^{2}}, \quad V_{\rho \eta}=-\frac{\eta}{\rho} \frac{1}{\sqrt{\rho^{2}+\eta^{2}}}
$$

and hence

$$
\rho^{2}\left(V_{\rho \eta}^{2}+V_{\eta \eta}^{2}\right)=1, \quad \text { which implies } u_{z}=\frac{2}{z},
$$

and the boundary condition is satisfied. So we can write a solution with $N$ such poles as

$$
\begin{gathered}
V=k_{0}^{3} \eta \log \rho+\sum_{i=1}^{N} k_{i}^{3} H_{i}(\rho, \eta), \\
H_{i}(\rho, \eta)=-\sqrt{\rho^{2}+\left(\eta-\eta_{i}\right)^{2}}+\left(\eta-\eta_{i}\right) \log \frac{\eta-\eta_{i}+\sqrt{\rho^{2}+\left(\eta-\eta_{i}\right)^{2}}}{\rho},
\end{gathered}
$$

where $\eta_{i}$ are the locations of the poles on the $\eta$ axis.

Interpreted as an electrostatics problem, this corresponds to the potential of a line charge along the $\eta$ axis of varying charge density $\lambda(\eta)$. The charge density profile $\lambda(\eta)$ is piecewise linear, with a "kink" at each $\eta_{i}$ as in figure 2 , such that

$$
\lambda^{\prime \prime}(\eta)=\sum_{i=1}^{N} k_{i}^{3} \delta\left(\eta-\eta_{i}\right) .
$$

where the parameters $k_{i}^{3}$ represent the amount by which the slope jumps as one moves across the kink at $\eta_{i}$ :

$$
\left.k_{i}^{3} \equiv \frac{d \lambda}{d \eta}\right|_{\eta_{i}+\epsilon}-\left.\frac{d \lambda}{d \eta}\right|_{\eta_{i}-\epsilon} .
$$

In $V$ (4.24), we have also put an additional parameter $k_{0}^{3}$, which represents the freedom to choose the value of $\lambda^{\prime}(\eta)$ at infinity. ${ }^{4}$

\footnotetext{
${ }^{4}$ Specifically, $2 k_{0}^{3}$ is the sum $\lambda^{\prime}(\infty)+\lambda^{\prime}(-\infty)$, while the difference $\lambda^{\prime}(\infty)-\lambda^{\prime}(-\infty)$ is given by the sum of all the jumps $k_{i}^{3}$.
} 


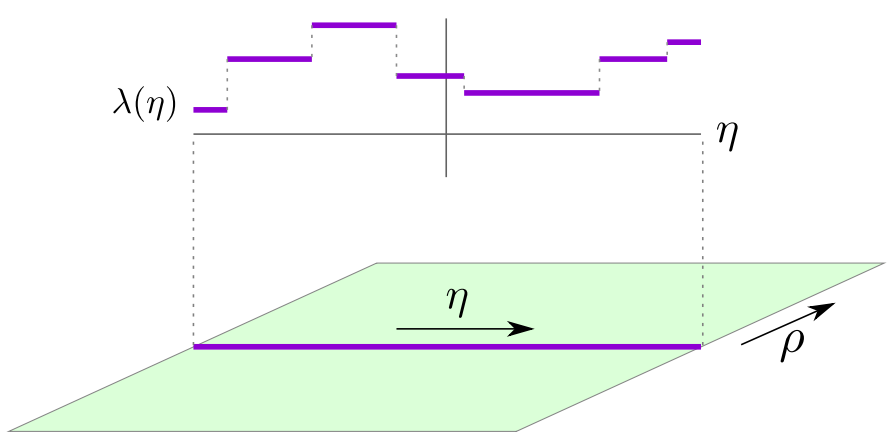

Figure 3. The electrostatics problem corresponding to $\hat{w}$. The line charge profile $\lambda(\eta)$ is piecewise constant, with "jumps" at each $\eta_{i}$.

We also choose $\hat{w}$ such that $w=\hat{w}_{z}$ has $1 / z$ type behavior at the Gibbons-Hawking points. It is easy to show that correct choice is

$$
\begin{gathered}
\hat{w}=q_{0} \log \rho+\sum_{i=1}^{N} q_{i} G_{i}(\rho, \eta), \\
G_{i}(\rho, \eta)=\log \frac{\eta-\eta_{i}+\sqrt{\rho^{2}+\left(\eta-\eta_{i}\right)^{2}}}{\rho} .
\end{gathered}
$$

As an electrostatics problem, this corresponds to a line charge profile $\lambda(\eta)$ which is piecewise constant, with "jumps" at each $\eta_{i}$ as in figure 3. The parameters $q_{i}$ give the amount of each jump:

$$
\left.q_{i} \equiv \lambda(\eta)\right|_{\eta_{i}+\epsilon}-\left.\lambda(\eta)\right|_{\eta_{i}-\epsilon}
$$

(where this $\lambda(\eta)$ is the one in figure 3 ).

For completeness, it is helpful to write out the $\eta$ - and $\rho$-derivatives of these, which appear in all other formulas:

$$
\begin{aligned}
V_{\eta \eta} & =\sum_{i=1}^{N} \frac{k_{i}^{3}}{\Sigma_{i}}, & V_{\rho \eta} & =\frac{k_{0}^{3}}{\rho}-\frac{1}{\rho} \sum_{i=1}^{N} \frac{k_{i}^{3}\left(\eta-\eta_{i}\right)}{\Sigma_{i}}, \\
\hat{w}_{\eta} & =\sum_{i=1}^{N} \frac{q_{i}}{\Sigma_{i}}, & \hat{w}_{\rho} & =\frac{q_{0}}{\rho}-\frac{1}{\rho} \sum_{i=1}^{N} \frac{q_{i}\left(\eta-\eta_{i}\right)}{\Sigma_{i}},
\end{aligned}
$$

where $\Sigma_{i} \equiv \sqrt{\rho^{2}+\left(\eta-\eta_{i}\right)^{2}}$. We note that this is essentially the same construction as in [38] for scalar-flat toric Kähler 4-manifolds (which can always be written in LeBrun form). Thus the base space is defined via the functions (4.31) and (4.32) and the $2 N+2$ parameters $k_{0}^{3}, k_{i}^{3}, q_{0}, q_{i}$.

\subsection{Near the singularities}

The base space is constructed out of $N$ points where the functions $V$ and $w$ are singular. In this section we look in the neighborhood of these points and show that the manifold is perfectly smooth, up to orbifold identifications, in a similar manner to Gibbons-Hawking 
metrics [9]. Specifically we will find that the metric (4.11) at these points locally approaches the orbifold $\mathbb{R}^{4} / G$, where $G \simeq \mathbb{Z}_{m} \times \mathbb{Z}_{n}$ is a finite subgroup of the maximal torus ${ }^{5} \mathrm{U}(1) \times$ $\mathrm{U}(1) \subset \mathrm{SO}(4)$.

Taking the limit as $(\rho, \eta) \rightarrow\left(0, \eta_{\ell}\right)$ for some $\eta_{\ell}$, we can define new coordinates

$$
\rho=R \sin \theta, \quad \eta-\eta_{\ell}=R \cos \theta .
$$

We will find it convenient to define the quantities

$$
\bar{K}_{\ell}^{3} \equiv \sum_{\substack{i \\ i \neq \ell}} k_{i}^{3} \operatorname{sign}\left(\eta_{\ell}-\eta_{i}\right), \quad \bar{Q}_{\ell} \equiv \sum_{\substack{i \\ i \neq \ell}} q_{i} \operatorname{sign}\left(\eta_{\ell}-\eta_{i}\right),
$$

and also the functions

$$
\begin{aligned}
\widetilde{K}(\theta) & \equiv\left(k_{\ell}^{3}\right)^{2}+\left(\bar{K}_{\ell}^{3}-k_{0}^{3}\right)^{2}+2 k_{\ell}^{3}\left(\bar{K}_{\ell}^{3}-k_{0}^{3}\right) \cos \theta, \\
\widetilde{Q}(\theta) & \equiv q_{\ell}^{2}+\left(\bar{Q}_{\ell}-q_{0}\right)^{2}+2 q_{\ell}\left(\bar{Q}_{\ell}-q_{0}\right) \cos \theta, \\
\widetilde{K Q}(\theta) & \equiv k_{\ell}^{3} q_{\ell}+\left(\bar{K}_{\ell}^{3}-k_{0}^{3}\right)\left(\bar{Q}_{\ell}-q_{0}\right)+\left(k_{\ell}^{3}\left(\bar{Q}_{\ell}-q_{0}\right)+q_{\ell}\left(\bar{K}_{\ell}^{3}-k_{0}^{3}\right)\right) \cos \theta .
\end{aligned}
$$

Then for small $R$, we have

$$
\rho^{2}\left(V_{\eta \eta}^{2}+V_{\rho \eta}^{2}\right) \rightarrow \widetilde{K}(\theta), \quad w \rightarrow \frac{1}{\widetilde{K}(\theta)} \frac{\widetilde{q}_{\ell}}{R}, \quad A \rightarrow-\frac{\widetilde{K Q}(\theta)}{\widetilde{K}(\theta)} \mathrm{d} \phi,
$$

where we define the determinant:

$$
\widetilde{q}_{\ell} \equiv q_{\ell}\left(\bar{K}_{\ell}^{3}-k_{0}^{3}\right)-k_{\ell}^{3}\left(\bar{Q}_{\ell}-q_{0}\right) .
$$

The metric becomes

$$
\mathrm{d} s^{2}=\frac{\widetilde{K}(\theta) R}{\widetilde{q}_{\ell}}\left(\mathrm{d} \tau-\frac{\widetilde{K Q}(\theta)}{\widetilde{K}(\theta)} \mathrm{d} \phi\right)^{2}+\frac{\widetilde{q}_{\ell}}{R}\left(\mathrm{~d} R^{2}+R^{2} \mathrm{~d} \theta^{2}\right)+\frac{\widetilde{q}_{\ell} R}{\widetilde{K}(\theta)} \sin ^{2} \theta \mathrm{d} \phi^{2},
$$

which, surprisingly enough, is flat. Setting $R=\varrho^{2} /\left(4 \widetilde{q}_{\ell}\right)$, this can be rearranged into the more convenient form

$$
\mathrm{d} s^{2}=\mathrm{d} \varrho^{2}+\frac{\varrho^{2}}{4}\left[\mathrm{~d} \theta^{2}+\frac{1}{\widetilde{q}_{\ell}^{2}}\left(\widetilde{K}(\theta) \mathrm{d} \tau^{2}-2 \widetilde{K Q}(\theta) \mathrm{d} \tau \mathrm{d} \phi+\widetilde{Q}(\theta) \mathrm{d} \phi^{2}\right)\right] .
$$

We compare this to a flat metric ${ }^{6}$ on $\mathbb{R}^{4}$ :

$$
\mathrm{d} s^{2}=\mathrm{d} \varrho^{2}+\frac{\varrho^{2}}{4}\left[\mathrm{~d} \theta^{2}+2(1+\cos \theta) \mathrm{d} \alpha^{2}+2(1-\cos \theta) \mathrm{d} \beta^{2}\right]
$$

where both $\alpha, \beta$ are (ordinarily) identified modulo $2 \pi$ and $\theta \in[0, \pi]$. The metrics (4.41) and (4.42) are then related by a coordinate transformation

$$
\tau=\left(q_{\ell}-\bar{Q}_{\ell}+q_{0}\right) \alpha-\left(q_{\ell}+\bar{Q}_{\ell}-q_{0}\right) \beta,
$$

\footnotetext{
${ }^{5}$ We note that the factors $\mathbb{Z}_{m}, \mathbb{Z}_{n} \subset \mathrm{U}(1) \times \mathrm{U}(1)$ are not necessarily rotations in a plane (i.e. fixing every point in the orthogonal plane). One can have, for example, $\mathbb{Z}_{m}$ acting in the first $\mathrm{U}(1)$ and $\mathbb{Z}_{n}$ acting in the diagonal $\mathrm{U}(1)$. Rotations in the diagonal $\mathrm{U}(1)$ fix only the origin.

${ }^{6}$ This metric is related to the standard spherical coordinates on $\mathbb{R}^{4}$ by $\theta=2 \vartheta$.
} 


$$
\phi=\left(k_{\ell}^{3}-\bar{K}_{\ell}^{3}+k_{0}^{3}\right) \alpha-\left(k_{\ell}^{3}+\bar{K}_{\ell}^{3}-k_{0}^{3}\right) \beta .
$$

To discover the precise geometry in the neighborhood of the origin, we must carefully follow the identifications of the angular coordinates. It is natural to identify the coordinates $(\tau, \phi)$ on the "diamond" lattice $\Gamma_{L B}$, given by the identifications

$$
(\tau, \phi): \quad(0,0) \sim(4 \pi, 0) \sim(2 \pi, 2 \pi) \sim(2 \pi,-2 \pi),
$$

whose basis can be written as a matrix $\Lambda_{L B}$ of column vectors which represent the coordinates where $(\tau, \phi)$ are identified:

$$
\Lambda_{L B}=2 \pi\left(\begin{array}{cc}
1 & 1 \\
1 & -1
\end{array}\right), \quad \text { or } \quad \Lambda_{L B}=2 \pi\left(\begin{array}{ll}
2 & 1 \\
0 & 1
\end{array}\right) .
$$

We are free to choose any pair of column vectors that generate the same lattice of identifications; alternatively, $\Lambda_{L B}$ is defined only up to right action by $G L(2, \mathbb{Z}) .^{7}$ Then applying the coordinate transformation (4.43) and (4.44), we find that the $(\alpha, \beta)$ coordinates should be identified on the lattice $\widetilde{\Gamma}$, generated by the basis

$$
\widetilde{\Lambda}=2 \pi \cdot \frac{1}{2 \widetilde{q}_{\ell}}\left(\begin{array}{cc}
k_{\ell}^{3}+\hat{K}_{\ell}^{3}+q_{\ell}+\hat{Q}_{\ell} & k_{\ell}^{3}+\hat{K}_{\ell}^{3}-q_{\ell}-\hat{Q}_{\ell} \\
k_{\ell}^{3}-\hat{K}_{\ell}^{3}+q_{\ell}-\hat{Q}_{\ell} & k_{\ell}^{3}-\hat{K}_{\ell}^{3}-q_{\ell}+\hat{Q}_{\ell}
\end{array}\right),
$$

where for ease of legibility we have defined

$$
\hat{K}_{\ell}^{3} \equiv \bar{K}_{\ell}^{3}-k_{0}^{3}, \quad \hat{Q}_{\ell} \equiv \bar{Q}_{\ell}-q_{0}
$$

We should then compare this lattice to a "reference" lattice $\Gamma$, generated by the basis

$$
\Lambda=2 \pi\left(\begin{array}{ll}
1 & 0 \\
0 & 1
\end{array}\right)
$$

which represents the ordinary $2 \pi$ identifications that $(\alpha, \beta)$ would take if there were no conical singularity. In order for the LeBrun metric to approach a proper orbifold $\mathbb{R}^{4} / G$ at the source point, one requires that the lattices $\Gamma, \widetilde{\Gamma}$ be compatible - that is, one must have that $\Gamma$ is a sublattice of $\widetilde{\Gamma}$. Otherwise, one has a conical point that is not an orbifold. ${ }^{8}$

The condition that $\Gamma \subseteq \widetilde{\Gamma}$ as lattices is equivalent to requiring that $\widetilde{\Lambda}^{-1} \Lambda$ be an integer matrix. We want this to be true at every $\eta_{\ell}$, in principle giving $N$ conditions; however, all of these conditions are equivalent to a single parity condition:

$$
\left(k_{0}^{3}+\sum_{i=1}^{N} k_{i}^{3}+q_{0}+\sum_{i=1}^{N} q_{i}\right) \in 2 \mathbb{Z},
$$

that is, the sum of all the parameters $k_{0}^{3}, k_{i}^{3}, q_{0}, q_{i}$ must be even. If we impose this condition, then at every $\eta_{\ell}$ the metric will approach an orbifold singularity.

\footnotetext{
${ }^{7}$ We define $G L(2, \mathbb{Z})$ as the group of $2 \times 2$ matrices with integer entries and determinant \pm 1 , hence invertible over $\mathbb{Z}$. This group is sometimes also called $S^{*} L(2, \mathbb{Z})$ or $S L^{ \pm}(2, \mathbb{Z})$.

${ }^{8} \mathrm{An}$ analogous situation with orbifolds of $\mathbb{R}^{2}$ is that the angular coordinate must be identified modulo $2 \pi / n$, but not modulo $2 \pi m / n$ for some $m>1$ ( $m, n$ relatively prime), as this would fail to be a quotient.
} 
At a given $\eta_{\ell}$, we can then compute the group $G$ that describes this orbifold singularity. The details are given in appendix A. The general procedure is as follows: given the lattices $\widetilde{\Gamma}, \Gamma$ generated by (4.47) and (4.49), one can find the group $G$ by reducing $\widetilde{\Lambda}^{-1} \Lambda$ to Smith normal form, where one diagonalizes $\widetilde{\Lambda}^{-1} \Lambda$ by left and right $G L(2, \mathbb{Z})$ actions:

$$
R=\widetilde{P}^{-1} \widetilde{\Lambda}^{-1} \Lambda P, \quad R=\left(\begin{array}{cc}
r_{1} & 0 \\
0 & r_{2}
\end{array}\right), \quad \text { where } \quad P, \widetilde{P} \in G L(2, \mathbb{Z}) .
$$

Given the parity condition (4.50), it is always true that $\widetilde{\Lambda}^{-1} \Lambda=2 \pi \widetilde{\Lambda}^{-1}$ has integer entries. Then the numbers $r_{1}, r_{2}$ are integers, and determine $G$ via

$$
G=\mathbb{Z}_{m} \times \mathbb{Z}_{n}, \quad \text { where } \quad m=r_{1}, \quad n=r_{2} .
$$

\subsubsection{Specific details of the groups $G$}

We then find a number of interesting facts (whose detailed derivation can be found in appendix A.2).

First, at every orbifold point one has, as mentioned, that $\Gamma \subseteq \widetilde{\Gamma}$ as a sublattice, and the group $G$ is formally given by the quotient $G \simeq \widetilde{\Gamma} / \Gamma$. The order of the group $G$ is

$$
\# G=\left|\operatorname{det}\left(\widetilde{\Lambda}^{-1} \Lambda\right)\right|=\left|\operatorname{det}\left(2 \pi \widetilde{\Lambda}^{-1}\right)\right|=\left|\widetilde{q}_{\ell}\right|,
$$

and thus the group $G$ is trivial exactly when $\widetilde{q}_{\ell}= \pm 1$. At such points, the metric approaches flat $\mathbb{R}^{4}$ with no conical singularity.

Second, we can ask when the orbifold point at $\eta_{\ell}$ is similar to the orbifold point of a charge $m>1$ Gibbons-Hawking metric. These are points where $G \simeq \mathbb{Z}_{m}$ and the action of $\mathbb{Z}_{m}$ is in the diagonal $\mathrm{U}(1)$ of the maximal torus $\mathrm{U}(1) \times \mathrm{U}(1) \in \mathrm{SO}(4)$. We find that such orbifold points occur whenever:

$$
\widetilde{q}_{\ell}= \pm m, \quad \frac{2\left(\bar{K}_{\ell}^{3}-k_{0}^{3}\right)}{\widetilde{q}_{\ell}} \in \mathbb{Z}, \quad \text { and } \quad \frac{2\left(\bar{Q}_{\ell}-q_{0}\right)}{\widetilde{q}_{\ell}} \in \mathbb{Z}
$$

One can also consider $G \simeq \mathbb{Z}_{m}$ acting in the anti-diagonal $\mathrm{U}(1)$, which results in similar conditions:

$$
\widetilde{q}_{\ell}= \pm m, \quad \frac{2 k_{\ell}^{3}}{\widetilde{q}_{\ell}} \in \mathbb{Z}, \quad \text { and } \quad \frac{2 q_{\ell}}{\widetilde{q}_{\ell}} \in \mathbb{Z}
$$

More generally, $G \simeq \mathbb{Z}_{m} \times \mathbb{Z}_{n}$ where each $\mathbb{Z}_{k}$ acts in some linear combination of the two U(1)'s. In the simplest case, the $\mathbb{Z}_{k}$ act by rotation within a plane; i.e. by rotating $\left(x^{1}, x^{2}\right)$ and leaving $\left(x^{3}, x^{4}\right)$ fixed. However, the "diagonal" rotations discussed above act in both planes and do not fix any point aside from the origin. One can also obtain more general rotations that rotate both $\left(x^{1}, x^{2}\right)$ and $\left(x^{3}, x^{4}\right)$ planes by unequal amounts.

In any case, an orbifold singularity with a finite group action such as $\mathbb{R}^{4} / G$ is benign in string theory [39], so in the context of microstate geometries, we will count such points as regular. 


\subsection{At infinity}

In the asymptotic region of the base metric, let us define

$$
\rho=R \sin \theta, \quad \eta=R \cos \theta .
$$

Then as $R \rightarrow \infty$, we have

$$
\begin{aligned}
\rho^{2}\left(V_{\rho \eta}^{2}+V_{\eta \eta}^{2}\right) & \rightarrow\left(k_{0}^{3}\right)^{2}+\left(K_{\star}^{3}\right)^{2}-2 k_{0}^{3} K_{\star}^{3} \cos \theta, \\
w & \rightarrow\left(\frac{q_{0} K_{\star}^{3}-k_{0}^{3} Q_{\star}}{\left(k_{0}^{3}\right)^{2}+\left(K_{\star}^{3}\right)^{2}-2 k_{0}^{3} K_{\star}^{3} \cos \theta}\right) \frac{1}{R}, \\
A & \rightarrow\left(\frac{k_{0}^{3} q_{0}+K_{\star}^{3} Q_{\star}-\left(q_{0} K_{\star}^{3}+k_{0}^{3} Q_{\star}\right) \cos \theta}{\left(k_{0}^{3}\right)^{2}+\left(K_{\star}^{3}\right)^{2}-2 k_{0}^{3} K_{\star}^{3} \cos \theta}\right) \mathrm{d} \phi,
\end{aligned}
$$

where the quantities $K_{\star}^{3}, Q_{\star}$ are defined as

$$
K_{\star}^{3} \equiv \sum_{i=1}^{N} k_{i}^{3}, \quad Q_{\star} \equiv \sum_{i=1}^{N} q_{i} .
$$

We see that (4.57)-(4.59) have the same structure as (4.38). So at infinity, the base metric approaches a metric with the same structure as (4.40). We can define the determinant

$$
\widetilde{q}_{\infty} \equiv q_{0} K_{\star}^{3}-k_{0}^{3} Q_{\star},
$$

and then the conditions (4.53) and (4.54), (4.55) apply in the same way. In particular, one has smooth $\mathbb{R}^{4}$ at infinity whenever

$$
\widetilde{q}_{\infty}= \pm 1 .
$$

One can obtain $\mathbb{R}^{4} / \mathbb{Z}_{m}$, where $\mathbb{Z}_{m}$ acts on the diagonal $\mathrm{U}(1)$ via

$$
\widetilde{q}_{\infty}= \pm m, \quad \frac{2 K_{\star}^{3}}{\widetilde{q}_{\infty}} \in \mathbb{Z}, \quad \text { and } \quad \frac{2 Q_{\star}}{\widetilde{q}_{\infty}} \in \mathbb{Z},
$$

or where $\mathbb{Z}_{m}$ acts on the anti-diagonal $\mathrm{U}(1)$ via

$$
\widetilde{q}_{\infty}= \pm m, \quad \frac{2 k_{0}^{3}}{\widetilde{q}_{\infty}} \in \mathbb{Z}, \quad \text { and } \quad \frac{2 q_{0}}{\widetilde{q}_{\infty}} \in \mathbb{Z} .
$$

In general, the geometry approaches $\mathbb{R}^{4} / G_{\infty}$, where again $G_{\infty} \simeq \mathbb{Z}_{m} \times \mathbb{Z}_{n}$.

\subsection{Ambipolar bases}

If the base space is considered in isolation, then we must restrict the "charges" $\widetilde{q}_{\ell}$ at each point to be positive. Otherwise, the function $w$ will change sign, ${ }^{9}$ and the signature of the metric (4.1) will flip from $(++++)$ to $(----)$.

However, in the context of supergravity solutions, the metric (4.1) appears multiplied by the warp factor $Z=\left(Z_{1} Z_{2} Z_{3}\right)^{1 / 3}$ in the full 5 -dimensional metric,

$$
\mathrm{d} s_{5}^{2}=-Z^{-2}(\mathrm{~d} t+k)^{2}+Z \mathrm{~d} s_{4}^{2} .
$$

\footnotetext{
${ }^{9}$ Caveat: this is not quite true, as we will show in section 4.6.
} 
Therefore, we can allow $w$ to change sign, so long as each of the $Z_{1}, Z_{2}, Z_{3}$ changes sign along the same locus, such that the 5 -dimensional metric retains the signature $(-++++)$. We call such a base space "ambipolar", where the signature is allowed to flip from $(++++)$ to $(----)$, as has been discussed at length in $[7,8]$. This justifies the use of $\widetilde{q}_{\ell}, \widetilde{q}_{\infty}= \pm 1, \pm m$ in (4.54), (4.55) and (4.62)-(4.64).

With this allowed flexibility in the charges $\widetilde{q}_{\ell}$, we can construct a wide variety of base spaces. In particular, it should be possible to have both $\widetilde{q}_{\ell}= \pm 1$ at every point and $\widetilde{q}_{\infty}= \pm 1$ at infinity, thus allowing us to write down supergravity solutions with an arbitrary number of bubbles and no orbifold points anywhere.

\subsection{Engineering solutions}

Here we will describe a simple algorithm for generating solutions with an arbitrary number of points $\eta_{\ell}$, each of which has trivial orbifold group (and thus is smooth). We will assume that each $\widetilde{q}_{\ell}=+1$ in order to show an interesting result. It is simple to generalize this algorithm to the more flexible ambipolar case where $\widetilde{q}_{\ell}= \pm 1$.

To derive this algorithm, we first observe that

$$
\bar{Q}_{i+1}-\bar{Q}_{i}=q_{i}+q_{i+1},
$$

and hence one has

$$
\left(\bar{Q}_{i+1}+q_{i+1}\right)=\left(\bar{Q}_{i}+q_{i}\right)+2 q_{i+1}
$$

and similarly for $\bar{K}_{i}^{3}$. The parity condition (4.50) can also be written

$$
k_{0}^{3}+q_{0}+\left(\bar{Q}_{i}+q_{i}\right)+\left(\bar{K}_{i}^{3}+k_{i}^{3}\right) \in 2 \mathbb{Z},
$$

where $i \in\{1 \ldots N\}$ is any of the $N$ points. Since the $q_{i}$ are integers, (4.67) guarantees that if (4.68) is true for any given $i$, it is true for all $i$. Therefore without explicitly writing down the sum of all the parameters, we can describe a recursive algorithm for constructing solutions starting at $i=1$ and adding as many points as we like.

A second observation we will need is that

$$
\begin{aligned}
\widetilde{q}_{i+1} \equiv q_{i+1}\left(\bar{K}_{i+1}^{3}-k_{0}^{3}\right)-k_{i+1}^{3}\left(\bar{Q}_{i+1}-q_{0}\right) \\
=q_{i+1}\left(\bar{K}_{i+1}^{3}+k_{i+1}^{3}-k_{0}^{3}\right)-k_{i+1}^{3}\left(\bar{Q}_{i+1}+q_{i+1}-q_{0}\right) \\
=q_{i+1}\left(\bar{K}_{i}^{3}+k_{i}^{3}+2 k_{i+1}^{3}-k_{0}^{3}\right) \\
\quad \quad-k_{i+1}^{3}\left(\bar{Q}_{i}+q_{i}+2 q_{i+1}-q_{0}\right) \\
\widetilde{q}_{i+1}=q_{i+1}\left(\bar{K}_{i}^{3}+k_{i}^{3}-k_{0}^{3}\right)-k_{i+1}^{3}\left(\bar{Q}_{i}+q_{i}-q_{0}\right),
\end{aligned}
$$

where the third line (4.71) follows from (4.67). Since we wish to set each $\widetilde{q}_{i}=1$, the last line (4.72) gives us a recurrence relation for the parameters $q_{i}, k_{i}^{3}$. Then the algorithm proceeds as follows:

1. Define

$$
a_{i} \equiv \bar{K}_{i}^{3}+k_{i}^{3}-k_{0}^{3}, \quad b_{i} \equiv \bar{Q}_{i}+q_{i}-q_{0},
$$

and choose any $a_{1}, b_{1}, k_{1}^{3}, q_{1}$ such that

$$
\widetilde{q}_{1} \equiv q_{1} a_{1}-k_{1}^{3} b_{1}=1, \quad a_{1}+b_{1}+k_{1}^{3}+q_{1} \in 2 \mathbb{Z} .
$$


2. Next, find some $k_{2}^{3}, q_{2}$ such that (using (4.72))

$$
\widetilde{q}_{2}=q_{2} a_{1}-k_{2}^{3} b_{1}=1,
$$

and such that

$$
a_{2}=a_{1}+2 k_{2}^{3}, \quad b_{2}=b_{1}+2 q_{2}
$$

are relatively prime. ${ }^{10}$

3. Repeat this as many times as desired, finding some $k_{i+1}^{3}, q_{i+1}$ such that

$$
\widetilde{q}_{i+1}=q_{i+1} a_{i}-k_{i+1}^{3} b_{i}=1,
$$

and

$$
a_{i+1}=a_{i}+2 k_{i+1}^{3}, \quad b_{i+1}=b_{i}+2 q_{i+1}
$$

are relatively prime.

4. After choosing $N$ such $k_{i}^{3}, q_{i}$, plug them all back into the definitions (4.73) along with $a_{1}, b_{1}$ from the initial step, and solve for the remaining parameters $k_{0}^{3}, q_{0}$.

It is simple to generalize this algorithm to produce a sequence of points with any desired $\widetilde{q}_{i}$. In this case, the requirement that each $a_{i}, b_{i}$ be relatively prime can be weakened, noting that in general, $\operatorname{gcd}\left(a_{i}, b_{i}\right)$ must divide both $\widetilde{q}_{i}$ and $\widetilde{q}_{i+1}$.

We also note that in the final step of the algorithm, there is no longer any freedom to choose parameters, and $k_{0}^{3}, q_{0}$ must be solved for, from (4.73). Therefore once we have laid down a sequence of $N$ points with given $\widetilde{q}_{i}$, the orbifold structure at infinity is fixed. ${ }^{11}$

If a specific behavior at infinity is required, one can re-write the algorithm to work backwards. The "reverse" algorithm is not identical to the one written here, but it is simple to work out from the reasoning in (4.67) and (4.68) along similar lines.

Using this algorithm it is easy to obtain some interesting solutions. We will give only the solutions and not the details of the algorithm used to obtain them. These two examples show some surprising features which emphasize the difference between LeBrun metrics and Gibbons-Hawking metrics regarding the types of allowed orbifold points:

\subsubsection{Example 1: every interior $\widetilde{q}_{i}=1$, but at infinity $\widetilde{q}_{\infty}=-1$}

The first example has three points, and is given by the parameters:

$$
\begin{aligned}
q_{1}=4, & q_{2}=-3, & q_{3}=2 ; & q_{0}=-2, \\
k_{1}^{3}=5, & k_{2}^{3}=-4, & k_{3}^{3}=1 ; & k_{0}^{3}=-1 .
\end{aligned}
$$

For this example, one has

$$
\widetilde{q}_{1}=1, \quad \widetilde{q}_{2}=1, \quad \widetilde{q}_{3}=1, \quad \widetilde{q}_{\infty}=-1 .
$$

\footnotetext{
${ }^{10}$ This is required in order for the next constraint $\widetilde{q}_{i+1}=1$ to have a solution.

${ }^{11}$ However, the orbifold structure at infinity depends on the specific $k_{i}^{3}, q_{i}$ of the solution, and the same sequence of $\widetilde{q}_{i}$ can result in different asymptotics!
} 
Hence at all the source points $\eta_{i}$ one has smooth $\mathbb{R}^{4}$ with trivial orbifold group. However, the minus sign in $\widetilde{q}_{\infty}$ reveals that it is possible for a LeBrun metric to flip signature $(++++)$ to $(----)$ at infinity even if all the interior points have positive "charges"!

This also implies that the naïve positivity condition mentioned at the beginning of section 4.5 is not quite correct, and requires that one also take into account the numerator of (4.58) to have a metric with positive signature everywhere. Since in the context of higher-dimensional supergravity solutions we do not require the signature of the base to remain $(++++)$ everywhere, we will not worry about this.

\subsubsection{Example 2: every interior $\widetilde{q}_{i} \geq 1$, but at infinity $\widetilde{q}_{\infty}=+1$}

A second important example is also given by three points:

$$
\begin{array}{llll}
q_{1}=-1, & q_{2}=2, & q_{3}=2 ; & q_{0}=2, \\
k_{1}^{3}=0, & k_{2}^{3}=1, & k_{3}^{3}=1 ; & k_{0}^{3}=1 .
\end{array}
$$

and this example has

$$
\widetilde{q}_{1}=3, \quad \widetilde{q}_{2}=1, \quad \widetilde{q}_{3}=1, \quad \widetilde{q}_{\infty}=1 .
$$

In this case the metric does not unexpectedly flip signature. However, we do see that it is possible for a LeBrun metric to be asymptotically flat (and not just locally flat) even if the interior "charges" are all positive and some of them are greater than 1 . This is in contrast to Gibbons-Hawking metrics, where it is a mathematical theorem that the only asymptotically (globally) flat hyper-Kähler metric in 4 dimensions is $\mathbb{R}^{4}$ [40]. Because LeBrun metrics are merely Kähler and not hyper-Kähler, they are not subject to this restriction, and the set of parameters (4.82) and (4.83) give an explicit example to this effect.

It does not, however, appear to be possible to choose parameters such that all the $\widetilde{q}_{i}=$ +1 and $\widetilde{q}_{\infty}=+1$, although we have not found a way to prove this impossibility in general.

\subsection{A topological ménagerie}

We have shown that the base metric approaches $\mathbb{R}^{4} / G$, for $G \simeq \mathbb{Z}_{m} \times \mathbb{Z}_{n}$, near each of the geometric charges where the $\tau$ fiber pinches off. As explained in section 3.1, these points control the appearance of homology 2-spheres as the $\tau$ fiber sweeps along a path between any two such points.

There are also additional phenomena which appear when we look more carefully at the axis in the 3 -dimensional base $h$ :

$$
\rho^{2}\left(V_{\rho \eta}^{2}+V_{\eta \eta}^{2}\right)\left(\mathrm{d} \rho^{2}+\mathrm{d} \eta^{2}\right)+\rho^{2} \mathrm{~d} \phi^{2}
$$

Along the axis, but away from the Gibbons-Hawking points, one has

$$
\rho^{2}\left(V_{\rho \eta}^{2}+V_{\eta \eta}^{2}\right) \rightarrow\left(k_{0}^{3}-\sum_{i=1}^{N} k_{i}^{3} \operatorname{sign}\left(\eta-\eta_{i}\right)\right)^{2} \equiv a^{2},
$$


which is a piecewise-constant function with jumps at each $\eta_{i}$. Whenever $a^{2}=1$, then as $\rho \rightarrow 0$, the $\phi$ circle pinches off smoothly. If instead $a^{2} \neq 1$ and $a^{2}>0$, then the $\phi$ circle pinches off in a conical singularity $\mathbb{R}^{2} / \mathbb{Z}_{a}$.

But it is also possible that $a=0$. Expanding to the next order in $\rho^{2}$, and imposing

$$
k_{0}^{3}=\sum_{i=1}^{N} k_{i}^{3} \operatorname{sign}\left(\eta-\eta_{i}\right)
$$

one has, as $\rho \rightarrow 0$,

$$
\rho^{2}\left(V_{\rho \eta}^{2}+V_{\eta \eta}^{2}\right) \rightarrow \rho^{2} f(\eta)^{2}, \quad w \rightarrow \frac{1}{\rho^{2}} \frac{g(\eta)}{f(\eta)^{2}}, \quad A \rightarrow-\frac{h(\eta)}{f(\eta)^{2}} \mathrm{~d} \phi,
$$

where the functions $f(\eta), g(\eta), h(\eta)$ are given by

$$
\begin{aligned}
f(\eta) & =\sum_{i=1}^{N} \frac{k_{i}^{3}}{\left|\eta-\eta_{i}\right|}, \\
g(\eta)= & \left(q_{0}-\sum_{i=1}^{N} q_{i} \operatorname{sign}\left(\eta-\eta_{i}\right)\right) f(\eta), \\
h(\eta)= & \sum_{i=1}^{N} \frac{q_{i}}{\left|\eta-\eta_{i}\right|} f(\eta) \\
& \quad+\frac{1}{2}\left(q_{0}-\sum_{i=1}^{N} q_{i} \operatorname{sign}\left(\eta-\eta_{i}\right)\right) \sum_{j=1}^{N} \frac{k_{j}^{3} \operatorname{sign}\left(\eta-\eta_{j}\right)}{\left(\eta-\eta_{j}\right)^{2}} .
\end{aligned}
$$

Then as $\rho \rightarrow 0$, the 4-metric can be rearranged to give

$$
g \rightarrow \frac{g(\eta)}{f(\eta)^{2}} \mathrm{~d} \phi+\frac{f(\eta)^{2}}{g(\eta)}\left[\frac{g(\eta)^{2}}{f(\eta)^{2}}\left(\mathrm{~d} \rho^{2}+\mathrm{d} \eta^{2}\right)+\rho^{2} \mathrm{~d} \tau^{2}\right]
$$

where the coordinates $\tau, \phi$ have now exchanged roles. Notably, along the entire segment over which $a$ (defined in (4.86)) vanishes, the $\phi$ circle remains a finite size as $\rho \rightarrow 0$, whereas the $\tau$ circle pinches off. In particular, we have

$$
\frac{g(\eta)^{2}}{f(\eta)^{2}}=\left(q_{0}-\sum_{i=1}^{N} q_{i} \operatorname{sign}\left(\eta-\eta_{i}\right)\right)^{2} \equiv 4 b^{2},
$$

so the $\tau$ circle is pinching off in a conical singularity $\mathbb{R}^{2} / \mathbb{Z}_{b}$ (the factor of 4 in (4.93) is to account for the fact that the period of $\tau$ is $4 \pi$ rather than $2 \pi$ ). This sort of homology 2 -cycle, in which $\phi$ remains finite while $\tau$ pinches off along a finite portion of the axis, is illustrated in figure 4.

We also point out that the axisymmetric LeBrun metrics we consider here are toric Kähler manifolds, and there is possibly a more elegant description of what is going on with the various types of 2-cycles using the techniques of toric geometry [38]. 


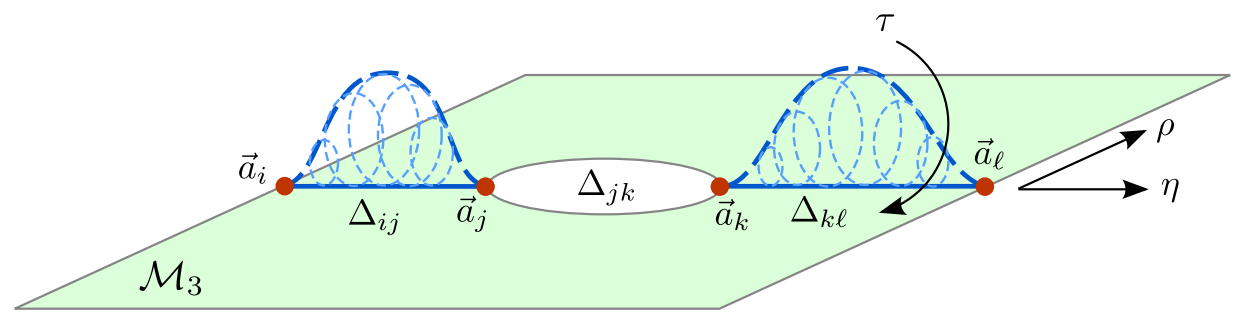

Figure 4. Homology 2-cycles in the axisymmetric base space. $\Delta_{i j}$ and $\Delta_{k \ell}$ are cycles formed by sweeping the $\tau$ fiber between source points. $\Delta_{j k}$ is a cycle formed by the $\phi$ circle. In the $\rho, \eta$ coordinates, the $\phi$-cycle appears as a line segment between $\vec{a}_{j}$ and $\vec{a}_{k}$. However, $\phi$ does not pinch off there, but approaches a finite size as $\rho \rightarrow 0$.

\subsection{Magnetic flux through cycles}

A desired property of these new solutions is that the magnetic 2-form $\Theta^{(3)}$ have non-trivial flux through the homological 2-cycles in the base. The 2 -form $\Theta^{(3)}$ is given by

$$
\Theta^{(3)}=\frac{1}{2}(\mathrm{~d} \tau+A) \wedge \mathrm{d} \frac{u_{z}}{w}+\frac{1}{2} w \underset{3}{*} \mathrm{~d} \frac{u_{z}}{w},
$$

but it will be more helpful to write it as

$$
\Theta^{(3)}=\mathrm{d} B^{3}=-\frac{1}{2} \mathrm{~d}\left[\frac{u_{z}}{w}(\mathrm{~d} \tau+A)+r u_{r} \mathrm{~d} \phi\right]
$$

where

$$
\begin{aligned}
\frac{1}{2} u_{z} & =\frac{V_{\eta \eta}}{\rho^{2}\left(V_{\rho \eta}^{2}+V_{\eta \eta}^{2}\right)}, \quad \frac{1}{2} r u_{r}=-1+\frac{1}{\rho\left(V_{\rho \eta}^{2}+V_{\eta \eta}^{2}\right)} V_{\rho \eta}, \\
w & =\frac{1}{\rho\left(V_{\rho \eta}^{2}+V_{\eta \eta}^{2}\right)}\left(V_{\eta \eta} \hat{w}_{\rho}-V_{\rho \eta} \hat{w}_{\eta}\right) .
\end{aligned}
$$

On a 2 -cycle $\Delta_{i j}$ swept out by the $\tau$ fiber, the flux can be computed via

$$
\Pi_{i j}^{(3)}=\frac{1}{4 \pi} \int_{\Delta_{i j}} \Theta^{(3)}=\frac{1}{4 \pi} \int_{\Delta_{i j}} \mathrm{~d} \tau \wedge \mathrm{d} \frac{K^{3}}{w}=\frac{k_{j}}{\widetilde{q}_{j}}-\frac{k_{i}}{\widetilde{q}_{i}},
$$

where $\widetilde{q}_{i} \equiv q_{i}\left(\bar{K}_{i}^{3}-k_{0}^{3}\right)-k_{i}^{3}\left(\bar{Q}_{i}-q_{0}\right)$. This is very reminiscent of the fluxes in the BPS case [7], and in stark contrast to previous non-BPS work [22, 23] where $\Theta^{(3)}$ had no topological fluxes.

On a 2-cycle formed by the $\phi$ circle, one has to be considerably more careful. Along a line segment of the $\eta$ axis between $\eta_{i}$ and $\eta_{j}$ where the $\phi$ circle has a finite size, one can show that as $\rho \rightarrow 0$,

$$
\Theta^{(3)} \rightarrow \frac{1}{g_{0}} \mathrm{~d}\left[-\mathrm{d} \tau+\frac{\tilde{f}(\eta)}{f(\eta)} \mathrm{d} \phi\right]
$$

where

$$
f(\eta)=\sum_{i=1}^{N} \frac{k_{i}^{3}}{\left|\eta-\eta_{i}\right|}, \quad \tilde{f}(\eta)=\sum_{i=1}^{N} \frac{q_{i}}{\left|\eta-\eta_{i}\right|}, \quad g_{0}=\left(q_{0}-\sum_{i=1}^{N} q_{i} \operatorname{sign}\left(\eta-\eta_{i}\right)\right)
$$


and we note that along this single line segment between two GH points, $g_{0}$ is constant. Outside this line segment, the approximation (4.99) no longer holds; in particular, we should not be concerned about the $\operatorname{sign}\left(\eta-\eta_{i}\right)$ in $g_{0}$, because the full $\Theta^{(3)}(4.94)$ is continuous everywhere and has no jumps. Then using (4.99), the flux of $\Theta^{(3)}$ through a $\phi$ cycle is given by

$$
\Pi_{i j}^{(3)}=\frac{1}{4 \pi} \int_{\Delta_{i j}} \frac{1}{g_{0}} \mathrm{~d} \frac{\tilde{f}(\eta)}{f(\eta)} \wedge \mathrm{d} \phi=\frac{1}{2 g_{0}}\left(\frac{q_{j}}{k_{j}^{3}}-\frac{q_{i}}{k_{i}^{3}}\right),
$$

which, interestingly, has a very different structure to (4.98).

Therefore we have succeeded in constructing a useful base space. It has the homological 2 -spheres we expected, swept out by $\tau$; these have cohomological fluxes which can be adjusted in any desired way by choosing parameters. As a bonus, we also obtain homological 2 -spheres swept out by $\phi$, which also have cohomological flux.

Interestingly, the fluxes of each type take different forms. If we assign units to the parameters of the solution, then $\tau$ fluxes have units of " $1 / q$ " and $\phi$ fluxes have units of " $1 / k$ ". This is consistent with the coordinate transformation (4.43), (4.44); if we assume the angles $\psi, \chi$ are dimensionless, then the the fluxes $\Pi_{i j}^{(3)}$ will have the same units through both $\tau$ cycles and $\phi$ cycles.

\section{Multi-centered supergravity solutions}

Now that we have an appropriate base space, we must solve the system (3.20), (3.21), (3.22), (3.24), (3.25), and finally (3.26). The route to the solutions is tedious and not particularly illuminating, so we refer the reader to appendix B for the details, including the full, explicit solutions themselves. In this section, we will focus on analyzing the solutions.

The solutions are described by $N$ number of points $\eta_{i}$ along the axis in the base space, and by the $8 N+10$ parameters $\left\{q_{0}, k_{0}^{1}, k_{0}^{2}, k_{0}^{3}, \ell_{1}^{0}, \ell_{2}^{0}, \ell_{3}^{0}, m_{0}, \omega_{0}, \ell_{3}^{z}, q_{i}, k_{i}^{1}, k_{i}^{2}, k_{i}^{3}, \ell_{1}^{i}, \ell_{2}^{i}, \ell_{3}^{i}, m_{i}\right\}$. The following sections make frequent reference to these parameters as they appear in the solutions of appendix B.

\subsection{Asymptotics of the 5d metric}

We should first look at the behavior of the 5-dimensional metric (2.4) at infinity. We leave the details to section B.1, and summarize the main results here.

We define the coordinates $R, \theta$ via

$$
\rho=R \sin \theta, \quad \eta=R \cos \theta,
$$

and look at the various metric functions as $R \rightarrow \infty$. First, we find that the warp factors $Z_{1}, Z_{2}$ go as $1 / R$ :

$$
Z_{1} \sim \frac{1}{R}, \quad Z_{2} \sim \frac{1}{R} .
$$

The functions $\mu, \omega_{(\phi)} \sim$ (const) at infinity, but to avoid CTC's, we must choose parameters such that these constants vanish. At the $1 / R$ order, these functions pick up an angular 
dependence $^{12}$ on $\theta$ :

$$
\mu \sim \frac{1}{R} f_{5}(\theta), \quad \omega_{(\phi)} \sim \frac{1}{R} f_{6}(\theta) .
$$

Next one is interested in $Z_{3}$, and one has a choice. The leading order behavior is constant:

$$
Z_{3} \sim \ell_{3}^{0}-\sum_{\substack{i j \\ i \neq j}} \frac{k_{i}^{1} \ell_{1}^{j}+k_{i}^{2} \ell_{2}^{j}-k_{i}^{3} \ell_{3}^{j}+2 m_{i} q_{j}}{\eta_{i}-\eta_{j}}+\mathcal{O}\left(\frac{1}{R}\right) .
$$

However, as mentioned in section 2, the $Z_{I}$ must all have the same asymptotic behavior to allow an M-theory lift. Hence we should choose $\ell_{3}^{0}$ to make the constant term vanish in (5.4). Alternatively, one can keep the constant term, allowing $Z_{3}$ to have different behavior to $Z_{1}, Z_{2}$ - as was pointed out in [23], this can be lifted naturally to the 6-dimensional theory obtained by reducing IIB supergravity on $T^{4}$.

\subsubsection{Asymptotics for lifting to 11d SUGRA}

We first consider the case that all three $Z_{I}$ have the same asymptotic behavior. Therefore the leading order constant $Z_{3}$ (B.19) must vanish, hence we set:

$$
\ell_{3}^{0}=\sum_{\substack{i j \\ i \neq j}} \frac{k_{i}^{1} \ell_{1}^{j}+k_{i}^{2} \ell_{2}^{j}-k_{i}^{3} \ell_{3}^{j}+2 m_{i} q_{j}}{\eta_{i}-\eta_{j}} .
$$

The 5-dimensional metric (2.4) then becomes

$$
\begin{aligned}
\mathrm{d} s_{5}^{2}=-\frac{R^{2}}{f_{4}(\theta)^{2}}\left[\mathrm{~d} t+\frac{1}{R} f_{5}(\theta) \mathrm{d} \tau+\frac{1}{R}\left(f_{5}(\theta) f_{3}(\theta)+f_{6}(\theta)\right) \mathrm{d} \phi\right]^{2} & \\
+ & \frac{f_{4}(\theta)}{f_{2}(\theta)}\left(\mathrm{d} \tau+f_{3}(\theta) \mathrm{d} \phi\right)^{2} \\
& \quad+\frac{f_{2}(\theta) f_{4}(\theta)}{R^{2}}\left[f_{1}(\theta)\left(\mathrm{d} R^{2}+R^{2} \mathrm{~d} \theta^{2}\right)+R^{2} \sin ^{2} \theta \mathrm{d} \phi^{2}\right],
\end{aligned}
$$

where generically speaking,

$$
\begin{aligned}
& \rho^{2}\left(V_{\rho \eta}^{2}+V_{\eta \eta}^{2}\right) \sim f_{1}(\theta), \quad w \sim \frac{1}{R} f_{2}(\theta), \quad A \sim f_{3}(\theta) \mathrm{d} \phi \\
& Z \sim \frac{1}{R} f_{4}(\theta), \quad \mu \sim \frac{1}{R} f_{5}(\theta), \quad \omega \sim \frac{1}{R} f_{6}(\theta) \mathrm{d} \phi,
\end{aligned}
$$

and simplifications likely occur in (5.6) if one works these out in more specificity. Due to the $\mathrm{d} R^{2} / R^{2}$ term, this metric is something related to $A d S_{2} \times S^{3}$. Specifically, it is a warped, rotating quotient $A d S_{2} \times S^{3} / G_{\infty}$, where $G_{\infty}$ is a finite group acting on the $S^{3}$ factor as described in section 4.4.

If we choose parameters such that $\widetilde{q}_{\infty}= \pm 1$ as defined in (4.61), then the base space approaches $\mathbb{R}^{4}$ without orbifold identifications, as described in section 4.5. One can then choose parameters such that

$$
Z_{3} \sim \frac{1}{R}, \quad \mu \sim \frac{1}{R}\left(c_{1}+c_{2} \cos \theta\right), \quad \omega \sim \mathcal{O}\left(R^{-2}\right),
$$

\footnotetext{
${ }^{12}$ The reason for labelling these functions " 5,6 " will become apparent in the next subsection.
} 
and therefore $Z \equiv\left(Z_{1} Z_{2} Z_{3}\right)^{1 / 3} \sim 1 / R$, without angular dependence. Then changing coordinates via

$$
R=\frac{1}{4} \varrho^{2}, \quad \theta=2 \vartheta, \quad \tau=\psi+\chi, \quad \phi=\psi-\chi
$$

(up to shifts in $t$ and $\tau$ ), one obtains a 5 -dimensional metric of the form

$$
\mathrm{d} s_{5}^{2}=-\varrho^{4}\left(\mathrm{~d} t+J_{1} \frac{\sin ^{2} \vartheta}{\varrho^{2}} \mathrm{~d} \psi+J_{2} \frac{\cos ^{2} \vartheta}{\varrho^{2}} \mathrm{~d} \chi\right)^{2}+\frac{\mathrm{d} \varrho^{2}}{\varrho^{2}}+\mathrm{d} \Omega_{3}^{2},
$$

which is the metric of the near-horizon region of a BMPV black hole [26].

\subsubsection{Asymptotics lifting to IIB on $T^{4}$}

Alternatively, we can choose to allow $Z_{3} \sim$ (const) at infinity while $Z_{1}, Z_{2} \sim 1 / \varrho^{2}$, and therefore not impose (5.5). Then the 5-dimensional metric will generically be of the form

$$
\mathrm{d} s_{5}^{2}=-\varrho^{8 / 3}(\mathrm{~d} t+k)^{2}+\varrho^{-4 / 3}\left(\mathrm{~d} \varrho^{2}+\varrho^{2} \mathrm{~d} \Omega_{3}^{2}\right),
$$

which looks somewhat strange. As shown in [23], however, there is a natural lift into 6dimensional $\mathcal{N}=1$ supergravity coupled to one anti-self-dual tensor multiplet [27-29]. The metric ansatz in 6 dimensions can be written in terms of the 5-dimensional quantities as

$$
\mathrm{d} s_{6}^{2}=-\frac{2}{\sqrt{Z_{1} Z_{2}}}\left(\mathrm{~d} v+B^{3}\right)\left(\mathrm{d} u+k-\frac{1}{2} Z_{3}\left(\mathrm{~d} v+B^{3}\right)\right)+\sqrt{Z_{1} Z_{2}} \mathrm{~d} s_{4}^{2},
$$

where $B^{3}$ is the 1 -form potential such at $\Theta^{(3)}=\mathrm{d} B^{3}$ as in (4.95). In this context, applying the asymptotics at infinity where $Z_{3} \sim$ (const) and $Z_{1}, Z_{2} \sim 1 / \varrho^{2}$ gives the result

$$
\mathrm{d} s_{6}^{2}=-2 \varrho^{2} \mathrm{~d} v\left(\mathrm{~d} u+k-\frac{1}{2} Z_{3} \mathrm{~d} v\right)+\frac{\mathrm{d} \varrho^{2}}{\varrho^{2}}+\mathrm{d} \Omega_{3}^{2},
$$

which is a momentum wave propagating on $A d S_{3} \times\left(S^{3} / G_{\infty}\right)$. Furthermore, nothing prevents us from imposing $Z_{3} \sim 1 / \varrho^{2}$ in this lifted metric; in such a case, one would obtain the 6-dimensional lift of the near-horizon BMPV metric (5.11), which is the near-horizon metric of a BPS, rotating D1-D5-P black string [41].

Summarizing asymptotics. Generally speaking, we see that our solutions are asymptotic to a warped, rotating version of $A d S_{2} \times\left(S^{3} / G_{\infty}\right)$, and for special choices of parameters, to near-horizon BMPV. Alternatively, one can lift to IIB supergravity on $T^{4}$, giving a 6-dimensional metric which allows $Z_{3}$ to have different asymptotics to $Z_{1}, Z_{2}$. In this case, one can impose $Z_{3} \sim$ (const) to obtain a momentum wave solution propagating on $A d S_{3} \times\left(S^{3} / G_{\infty}\right)$; or, imposing $Z_{3} \sim 1 / \varrho^{2}$, one obtains the near-horizon metric of a BPS, rotating black string.

We should note from constraints derived in [22], that the "floating brane" equations [20] on a Kähler base do not have asymptotically flat solutions, and solutions must generically have nonzero rotation parameters at infinity. The reason for this is that the $T_{00}$ component of the 5-dimensional energy-momentum tensor is a manifestly positive-definite function of the $Z_{I}, \Theta^{(I)}$. If we have $Z_{I} \sim 1$ at infinity, then $\Theta^{(1)}, \Theta^{(2)}$ still contain a term proportional to the Kähler form $J$, which contributes a constant to $T_{00}$ and prevents asymptotic flatness. The rotation at infinity comes from the off-diagonal terms $T_{0 a}$, which also do not vanish. 


\subsection{Regularity conditions}

The solutions we have obtained generically have a number of singularities at each $\eta_{i}$ which act as sources of the electric potentials $Z_{I}$ and magnetic field strengths $\Theta^{(I)}$. However, in the context of black hole microstate geometries, we are interested in solutions that are everywhere smooth, with no singular sources. This can be accomplished by choosing the parameters in such a way that singularities are eliminated. The necessary condition for smoothness is that each of the functions $Z_{1}, Z_{2}, Z_{3}, \mu, \omega_{(\phi)}$ remain non-singular as the GH points are approached.

Looking near a point $\eta_{\ell}$, we again define a local radial coordinate via

$$
\rho=R \sin \theta, \quad \eta-\eta_{\ell}=R \cos \theta .
$$

Then as $R \rightarrow 0$, we have

$$
\begin{aligned}
& Z_{1} \rightarrow \frac{1}{R}\left(\frac{k_{\ell}^{2} k_{\ell}^{3}+q_{\ell} \ell_{1}^{\ell}}{q_{\ell}\left(\bar{K}_{\ell}^{3}-k_{0}^{3}\right)-k_{\ell}^{3}\left(\bar{Q}_{\ell}-q_{0}\right)}\right), \\
& Z_{2} \rightarrow \frac{1}{R}\left(\frac{k_{\ell}^{1} k_{\ell}^{3}+q_{\ell} \ell_{2}^{\ell}}{q_{\ell}\left(\bar{K}_{\ell}^{3}-k_{0}^{3}\right)-k_{\ell}^{3}\left(\bar{Q}_{\ell}-q_{0}\right)}\right),
\end{aligned}
$$

where again,

$$
\bar{K}_{\ell}^{3} \equiv \sum_{\substack{i \\ i \neq \ell}} k_{i}^{3} \operatorname{sign}\left(\eta_{\ell}-\eta_{i}\right), \quad \bar{Q}_{\ell} \equiv \sum_{\substack{i \\ i \neq \ell}} q_{i} \operatorname{sign}\left(\eta_{\ell}-\eta_{i}\right) .
$$

Therefore, the singular parts of $Z_{1}, Z_{2}$ will vanish if

$$
\ell_{1}^{\ell}=-\frac{k_{\ell}^{2} k_{\ell}^{3}}{q_{\ell}}, \quad \ell_{2}^{\ell}=-\frac{k_{\ell}^{1} k_{\ell}^{3}}{q_{\ell}},
$$

at every GH point. Next, imposing (5.19), we have

$$
\begin{gathered}
Z_{3} \rightarrow \frac{1}{R}\left[\frac{k_{\ell}^{1} k_{\ell}^{2}}{q_{\ell}^{2}}\left(q_{\ell}\left(\bar{K}_{\ell}^{3}-k_{0}^{3}\right)-k_{\ell}^{3}\left(\bar{Q}_{\ell}-q_{0}\right)\right)-\ell_{3}^{\ell}\left(\bar{K}_{\ell}^{3}-k_{0}^{3}\right)+2 m_{\ell}\left(\bar{Q}_{\ell}-q_{0}\right)\right. \\
\left.-\left(k_{\ell}^{3} \ell_{3}^{\ell}+2 m_{\ell} q_{\ell}\right) \cos \theta\right]
\end{gathered}
$$

and hence the singular part of $Z_{3}$ vanishes if

$$
\ell_{3}^{\ell}=\frac{k_{\ell}^{1} k_{\ell}^{2}}{q_{\ell}}, \quad m_{\ell}=-\frac{k_{\ell}^{1} k_{\ell}^{2} k_{\ell}^{3}}{2 q_{\ell}^{2}} .
$$

Together, (5.19) and (5.21) are also sufficient to guarantee $\mu \rightarrow$ (const) and $\omega_{(\phi)} \rightarrow$ (const) near $\eta_{\ell}$; hence we will have a regular solution if we impose these conditions at every GH point.

We note that these conditions appear exactly the same (up to signs that result from differing conventions) as those in the original BPS story [7]. However, there is a key difference: in these solutions, the parameters $q_{\ell}$ do not directly control the singularities of $w$, but as in (4.38), the singularities in $w$ are controlled by the determinants

$$
\widetilde{q}_{\ell} \equiv q_{\ell}\left(\bar{K}_{\ell}^{3}-k_{0}^{3}\right)-k_{\ell}^{3}\left(\bar{Q}_{\ell}-q_{0}\right) .
$$




\subsection{Fluxes through cycles}

It will be useful to have expressions for the magnetic flux threading 2-cycles formed by sweeping the $\tau$ fiber between GH points in the 4-dimensional base space. We have already calculated the flux of $\Theta^{(3)}$ on these cycles (4.98):

$$
\Pi_{i j}^{(3)} \equiv \frac{1}{4 \pi} \int_{\Delta_{i j}} \Theta^{(3)}=\frac{k_{j}}{\widetilde{q}_{j}}-\frac{k_{i}}{\widetilde{q}_{i}} .
$$

Before computing the remaining two fluxes, we will impose the regularity conditions (5.19), (5.21). Then as we approach a GH point $\eta_{\ell}$, we have

$$
\frac{K^{1}}{w} \rightarrow \frac{k_{\ell}^{1}\left(\bar{K}_{\ell}^{3}-k_{0}^{3}\right)}{q_{\ell}}-\ell_{2}^{0}+\bar{L}_{2}^{\ell}, \quad \frac{K^{2}}{w} \rightarrow \frac{\left.k_{\ell}^{2} \overline{(} K_{\ell}^{3}-k_{0}^{3}\right)}{q_{\ell}}-\ell_{1}^{0}+\bar{L}_{1}^{\ell},
$$

where we have defined new quantities

$$
\bar{L}_{1}^{\ell} \equiv \sum_{\substack{i \\ i \neq \ell}} \ell_{1}^{i} \operatorname{sign}\left(\eta_{\ell}-\eta_{i}\right), \quad \bar{L}_{2}^{\ell} \equiv \sum_{\substack{i \\ i \neq \ell}} \ell_{2}^{i} \operatorname{sign}\left(\eta_{\ell}-\eta_{i}\right) .
$$

Then the flux through $\tau$ cycles can be computed in a way similar to (4.98):

$$
\begin{aligned}
& \Pi_{i j}^{(1)} \equiv \frac{1}{4 \pi} \int_{\Delta_{i j}} \Theta^{(1)}=\frac{k_{j}^{1}\left(\bar{K}_{j}^{3}-k_{0}^{3}\right)}{q_{j}}+\bar{L}_{2}^{j}-\frac{k_{i}^{1}\left(\bar{K}_{i}^{3}-k_{0}^{3}\right)}{q_{i}}-\bar{L}_{2}^{i}, \\
& \Pi_{i j}^{(2)} \equiv \frac{1}{4 \pi} \int_{\Delta_{i j}} \Theta^{(2)}=\frac{k_{j}^{2}\left(\bar{K}_{j}^{3}-k_{0}^{3}\right)}{q_{j}}+\bar{L}_{1}^{j}-\frac{k_{i}^{2}\left(\bar{K}_{i}^{3}-k_{0}^{3}\right)}{q_{i}}-\bar{L}_{1}^{i} .
\end{aligned}
$$

One can in principle also compute the fluxes through the 2-cycles swept out by $\phi$, as was done in section 4.8. However, this is tedious and of no special benefit to the rest of this analysis, so we omit it.

\subsection{Causality conditions: the "bubble equations"}

We have determined the conditions that a solution is smooth as one approaches the various Gibbons-Hawking points in the base manifold. However, to construct sensible supergravity solutions, one must also ensure that there are no closed timelike curves.

Looking at the metric (2.4) on a surface of constant $t$, we can rearrange it as follows:

$$
\begin{gathered}
\mathrm{d} s_{5}^{2}=\frac{\mathcal{Q}}{w^{2} Z^{2}}\left(\mathrm{~d} \tau+A-\frac{w^{2} \mu}{\mathcal{Q}} \omega\right)^{2}+Z w\left(\rho^{2} \mathrm{~d} \phi^{2}-\frac{\omega^{2}}{\mathcal{Q}}\right) \\
+Z w \rho^{2}\left(V_{\rho \eta}^{2}+V_{\eta \eta}^{2}\right)\left(\mathrm{d} \rho^{2}+\mathrm{d} \eta^{2}\right)
\end{gathered}
$$

where

$$
\mathcal{Q} \equiv Z_{1} Z_{2} Z_{3} w-w^{2} \mu^{2}, \quad Z \equiv\left(Z_{1} Z_{2} Z_{3}\right)^{1 / 3} .
$$

In order for CTC's to be absent everywhere, (5.28) must be positive-definite. This requires

$$
\mathcal{Q} \geq 0, \quad Z w \geq 0, \quad \rho^{2} \mathrm{~d} \phi^{2} \geq \frac{\omega^{2}}{\mathcal{Q}} .
$$


It is generally impractical to enforce these global conditions from the local point of view of choosing parameters in the solution; one must write down a solution and then explore it numerically to look for CTC's. However, one can look at local causality conditions near the GH points, and this leads to a system of equations that must be solved as a necessary (but not sufficient) condition that a solution be causally sensible.

In the BPS context [7], this leads to a system of so-called "bubble equations" that relate the distances between the $\mathrm{GH}$ centers (as measured in the $\mathbb{R}^{3}$ ) to the product of the fluxes of the $\Theta^{(I)}$ through the various 2-cycles described by the GH centers. Thus the size of each "bubble" is governed by the amount of flux trapped on it. Importantly, the bubble equations depend upon the product of all three fluxes. In previous work on non-supersymmetric solutions derived from floating branes [22, 23], the third flux $\Theta^{(3)}$ was topologically trivial and contributed no fluxes to the bubble equations. The result was that the causality conditions did not constrain the sizes of the homological 2-cycles. In these new solutions, however, $\Theta^{(3)}$ has non-trivial fluxes on the 2-cycles (as in section 4.8), so we expect to find non-trivial bubble equations.

Looking at (5.28) near the GH points, one finds two potential sources of CTC's coming from the two angular coordinates $\tau, \phi$. To eliminate CTC's near the GH points, we must require that

$$
\mu \rightarrow 0, \quad \omega \rightarrow 0
$$

at these points. While these appear to be two different conditions, they are really the same. To see this, we can rearrange the $\omega$ equation (3.26) as follows:

$$
\begin{aligned}
& \mathrm{d} \omega=w Z_{1} \star \mathrm{d} \frac{K^{1}}{w}+w Z_{2} \star \frac{\mathrm{d}}{3} \frac{K^{2}}{w}+w Z_{3} \star \mathrm{d} \frac{K^{3}}{w}-2 w Z_{1} Z_{2} \underset{3}{ } \mathrm{~d} z \\
& +w \star x \mathrm{~d} \mu-\mu \mathrm{d} A \text {. }
\end{aligned}
$$

We choose parameters such that $\omega$ vanishes at infinity (as in (B.22), (B.23)), so for $\omega$ to be non-vanishing somewhere on the axis would require Dirac-Misner strings. Given the regularity conditions (5.19), (5.21), the only term in (5.32) that can source Dirac-Misner strings is $-\mu \mathrm{d} A$. Therefore, to eliminate local CTC's near the GH points, it is enough to demand that $\mu$ vanish at each GH point. The vanishing of $\mu$ results in the following "bubble equations" at each $\eta_{\ell}$ :

$$
\begin{aligned}
-2 m_{0} \widetilde{q}_{\ell}+k_{0}^{3} \ell_{3}^{0}=\left(k_{0}^{3}-\right. & \left.\bar{K}_{\ell}^{3}\right) \sum_{\substack{i \\
i \neq \ell}} \widehat{\Pi}_{\ell i}^{(1)} \widehat{\Pi}_{\ell i}^{(2)} \widehat{\Pi}_{\ell i}^{(3)} \frac{q_{\ell} q_{i}}{r_{\ell i}} \\
& +\frac{1}{2} k_{\ell}^{3} \sum_{\substack{i j \\
i \neq j}} \widehat{\Pi}_{i j}^{(1)} \widehat{\Pi}_{i j}^{(2)} \widehat{\Pi}_{i j}^{(3)} \frac{q_{i} q_{j}}{r_{i j}} s(i, j) s(\ell, i) s(\ell, j)
\end{aligned}
$$

where we have defined

$$
\begin{aligned}
r_{i j} \equiv\left|\eta_{i}-\eta_{j}\right|, \quad \widehat{\Pi}_{i j}^{(I)} & \equiv\left(\frac{k_{j}^{I}}{q_{j}}-\frac{k_{i}^{I}}{q_{i}}\right), \quad s(a, b) \equiv \operatorname{sign}\left(\eta_{a}-\eta_{b}\right), \\
\widetilde{q}_{\ell} & \equiv q_{\ell}\left(\bar{K}_{\ell}^{3}-k_{0}^{3}\right)-k_{\ell}^{3}\left(\bar{Q}_{\ell}-q_{0}\right) .
\end{aligned}
$$


The combinations of parameters $\widehat{\Pi}_{i j}^{(I)}$ which appear in the bubble equations are not the physical fluxes $\Pi_{i j}^{(I)}$ calculated in (4.98), (5.26) and (5.27). However, with a little algebra one can show that they are related linearly and homogeneously: ${ }^{13}$

$$
\begin{gathered}
\Pi_{\ell i}^{(1)}=\left(-k_{0}^{3}+\bar{K}_{\ell}^{3}\right) \widehat{\Pi}_{\ell i}^{(1)}+\sum_{j=1}^{N} k_{j}^{3} \widehat{\Pi}_{i j}^{(1)}(s(\ell, j)-s(i, j)), \\
\Pi_{\ell i}^{(2)}=\left(-k_{0}^{3}+\bar{K}_{\ell}^{3}\right) \widehat{\Pi}_{\ell i}^{(2)}+\sum_{j=1}^{N} k_{j}^{3} \widehat{\Pi}_{i j}^{(2)}(s(\ell, j)-s(i, j)), \\
\widetilde{q}_{\ell} \widetilde{q}_{i} \Pi_{\ell i}^{(3)}=q_{\ell} q_{i}\left(-k_{0}^{3}+\bar{K}_{\ell}^{3}\right) \widehat{\Pi}_{\ell i}^{(3)}+k_{\ell}^{3} \sum_{j=1}^{N} q_{i} q_{j} \widehat{\Pi}_{i j}^{(3)}(s(\ell, j)-s(i, j)) .
\end{gathered}
$$

These look tantalizingly like they might allow a simpler expression of the right-hand side of (5.33); however, the presence of $1 / r_{\ell i}, 1 / r_{i j}$ in the sums complicates the algebra, and the expression we have written in (5.33) is probably the simplest.

We have thus succeeded in finding a family of non-BPS solutions with non-trivial bubble equations which constrain the bubble diameters $r_{i j}$ in terms of the fluxes trapped on the bubbles. We also observe that there is a significant, important difference between these non-BPS bubble equations and the well-known BPS version [7]. The term on the second line of (5.33) is entirely new: in order to avoid CTC's at $\eta_{\ell}$, the equations depend not only on the diameters $r_{\ell i}$ of the 2-cycles adjacent to $\eta_{\ell}$, but also on the diameters $r_{i j}$ of each of the other 2-cycles. This is telling us about new physics: these non-supersymmetric solutions exhibit a richer variety of $E \times B$ interactions than previously known BPS solutions.

However, while these bubble equations differ from the BPS ones in a few ways, they are similar in a particularly striking way: they are linear in the inverse bubble diameters $1 / r_{i j}$. This stands in contrast to the so-called "almost BPS" family of solutions where the bubble equations are cubic in the inverse distances [16-18]. So although these solutions lack supersymmetry, they are in some sense closer to BPS than the "almost BPS" solutions. This is because they are trivial KK reductions of 6-dimensional geometries which are BPS in the IIB frame [23].

Number of independent bubble equations. Ultimately, there are only $N-1$ independent $r_{i j}$, so we expect there to be $N-1$ independent bubble equations. This is easiest to demonstrate by looking directly at the Dirac-Misner strings in $\omega$. This results in the same bubble equations as above, each multiplied by a constant (which is different at each $\eta_{\ell}$ ). Near $\eta_{\ell}$, the Dirac-Misner string part of $\omega$ is given by the jump that occurs in crossing from one side of $\eta_{\ell}$ to the other:

$$
\left.\omega\right|_{\theta=0}-\left.\omega\right|_{\theta=\pi}=-\left(\left.A\right|_{\theta=0}-\left.A\right|_{\theta=\pi}\right) \mu=\frac{2 \widetilde{q}_{\ell}}{\left(\bar{K}_{\ell}^{3}-k_{0}^{3}\right)^{2}-\left(k_{\ell}^{3}\right)^{2}} \mu \mathrm{d} \phi .
$$

Since $\omega$ contains a sequence of Dirac-Misner string sources along the $\eta$ axis, and vanishes at both positive and negative infinity, then the sum of all the jumps must be zero. Therefore,

\footnotetext{
${ }^{13}$ Here we again assume the regularity conditions (5.19), (5.21) are imposed.
} 
the weighted sum of all the bubble equations (5.33), each multiplied by the coefficient in (5.39), must give zero. This weighted sum gives

$$
m_{0}=\frac{1}{2} \frac{K_{\star}^{3}}{q_{0} K_{\star}^{3}-k_{0}^{3} Q_{\star}}\left(\ell_{3}^{0}-\sum_{\substack{i j \\ i \neq j}} \frac{k_{i}^{1} \ell_{1}^{j}+k_{i}^{2} \ell_{2}^{j}-k_{i}^{3} \ell_{3}^{j}+2 m_{i} q_{j}}{\eta_{i}-\eta_{j}}\right) .
$$

which is the condition we have already imposed (B.22) in order that $\mu \rightarrow 0$ at infinity. Hence as expected, the bubble equations constitute $N-1$ independent equations in the $N-1$ independent variables $r_{i j}$.

Hints of scaling solutions. Finally, there is a curious thing that happens if we impose all of the conditions derived in section 5.1 for near-horizon BMPV-like (i.e. warped, rotating $\left.A d S_{2} \times S^{3}\right)$ asymptotics. First we note that the value of $\ell_{3}^{0}$ in (5.5) is entirely a linear combation of the inverse bubble diameters $1 / r_{i j}$. Second, when (5.5) is imposed, then $m_{0}=\omega_{0}=0$ as in (B.22), (B.23). Therefore if we insist on near-horizon BMPV-like asymptotics, the bubble equations will take the form, schematically,

$$
\sum \widehat{\Pi}^{(1)} \widehat{\Pi}^{(2)} \widehat{\Pi}^{(3)} \frac{q q}{r}=0 .
$$

If we instead think of this equation as a limiting process where we replace the right-hand side with some $\delta$ and let $\delta \rightarrow 0$, then the solutions, as we follow this process, are scaling solutions $[17,42,43]$. The right-hand side roughly scales as $(\Pi)^{3} / r$, and thus if we adjust the dipole charges while simultaneously shrinking the bubble diameters, such that $\Pi \sim \lambda, r \sim \lambda$ for $\lambda$ small, this tends toward zero. In such solutions, the overall size of the bubbled region shrinks (as measured in the 3-dimensional base), while the ratios between the bubble sizes becomes constant. In the full 5 -dimensional metric, this represents the appearance of an arbitrarily deep throat, smoothly capped off by topological bubbles at some finite depth. Thus one can see the near-horizon BMPV geometry, and the related rotating-AdS-like metrics with angular dependence as in (5.6), as the result of this limiting procedure.

More generally, if we consider asymptotic conditions where $Z_{3}$ behaves differently from $Z_{1}, Z_{2}$ (thus naturally lifting to the $6 \mathrm{~d}$ IIB metric (5.14) rather than to $11 \mathrm{~d}$ supergravity), we can set the constant $\ell_{3}^{0}$ to anything we like. In this case, one can find finite, non-trivial solutions to the bubble equations without subjecting them to a limiting procedure. We demonstrate this in section 5.5.

\subsection{An explicit numerical example}

In this section we will give an explicit, solved example with three source points, illustrating how a smooth, CTC-free solution can be constructed. The solution will be in the class asymptotic to (5.14), where $Z_{3} \sim$ (const) and $Z_{1} \sim 1 / \rho^{2}, Z_{2} \sim 1 / \rho^{2}$. We will focus on satisfying the local conditions near the points, and not delve into exactly what asymptotics result.

We begin by choosing three source points along the $\eta$ axis and assigning them geometric charges. The parameters of the solution are ordered in the manner drawn in figure 5; thus 


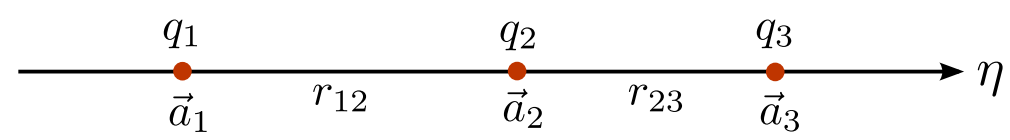

Figure 5. Setup for a 3-center example. Geometric charges $q_{1}, q_{2}, q_{3}$ are put at the points $\vec{a}_{1}, \vec{a}_{2}, \vec{a}_{3}$ along the $\eta$ axis. One must then solve the bubble equations to find $r_{12}, r_{23}$.

by hypothesis the bubble diameters $r_{12}, r_{23}$ are positive. At the points $\vec{a}_{1}, \vec{a}_{2}, \vec{a}_{3}$ we put the following charges:

$$
\begin{array}{rlrlrl}
q_{0} & =2, & q_{1} & =3, & q_{2} & =2, \\
k_{0}^{1} & =0, & k_{1}^{1}=5, & k_{2}^{1}=2, & q_{3}=6, \\
k_{0}^{2}=0, & k_{1}^{2}=5, & k_{2}^{2}=4, & k_{3}^{2}=3, \\
k_{0}^{3}=1, & k_{1}^{3}=2, & k_{2}^{3}=2, & k_{3}^{3}=2, \\
\ell_{1}^{0}=0, & \ell_{2}^{0}=0, & \ell_{3}^{0}=10, & \ell_{3}^{z}=0 .
\end{array}
$$

Our particular choices are made to satisfy a few constraints: 1) the parity condition (4.50) such that each point will be an orbifold point; 2) the condition that all the $\widehat{\Pi}_{i j}^{(I)}$ are nonzero; 3 ) the condition that the $\widetilde{q}_{i}$ are all "nice" numbers; 4) the condition that the bubble equations yield real, positive solutions for the $r_{i j}$; and 5) the condition that $\mathcal{Q}>0$ in order to be free of CTC's. Choosing parameters (5.42) to satisfy all of these properties is a bit of an art, and it would be interesting to better understand the moduli space of physical solutions.

The value of $\ell_{3}^{0}$ sets the overall scale of the solution, as it is the only unconstrained constant sitting on the left-hand side of (5.33). Since we have put $\ell_{3}^{0} \neq 0$, this solution will have asymptotics best described in the $6 \mathrm{~d}$ IIB frame as in (5.14). Most of the functions $w, K^{I}, L_{I}, M$ that make up the solution are too lengthy to write out, but as an example, we have

$$
\begin{aligned}
& \hat{w}_{\eta}=\frac{3}{\sqrt{\rho^{2}+\eta^{2}}}+\frac{2}{\sqrt{\rho^{2}+\left(\eta-r_{12}\right)^{2}}}+\frac{6}{\sqrt{\rho^{2}+\left(\eta-r_{12}-r_{23}\right)^{2}}}, \\
& \hat{w}_{\rho}=\frac{2}{\rho}-\frac{3 \eta}{\rho \sqrt{\rho^{2}+\eta^{2}}}-\frac{2\left(\eta-r_{12}\right)}{\rho \sqrt{\rho^{2}+\left(\eta-r_{12}\right)^{2}}}-\frac{6\left(\eta-r_{12}-r_{23}\right)}{\rho \sqrt{\rho^{2}+\left(\eta-r_{12}-r_{23}\right)^{2}}},
\end{aligned}
$$

and so on. There are two remaining constants $m_{0}, \omega_{0}$ which we have not set in (5.42). To meet the regularity conditions at infinity, these constants will be set equal to (B.22) and (B.23), and then their numerical values will be determined after the $r_{i j}$ are known via solving the bubble equations (5.33).

At each source point, the base metric approaches $\mathbb{R}^{4} / G_{\ell}$, where the order of $G_{\ell}$ at the source point $\eta_{\ell}$ is given by $\# G_{\ell}=\left|\widetilde{q}_{\ell}\right|$, and for the parameters (5.42) these $\widetilde{q}_{\ell}$ are given by

$$
\widetilde{q}_{1}=5, \quad \widetilde{q}_{2}=8, \quad \widetilde{q}_{3}=12, \quad \widetilde{q}_{\infty}=1 .
$$

Therefore we see that this is another example of the phenomenon described in section 4.6, where the base metric can be asymptotically globally flat, despite having orbifold points on the interior, and without resorting to making it "ambipolar" as described in section 4.5. 
1) $G_{1} \simeq \mathbb{Z}_{5}^{\text {diag }}$

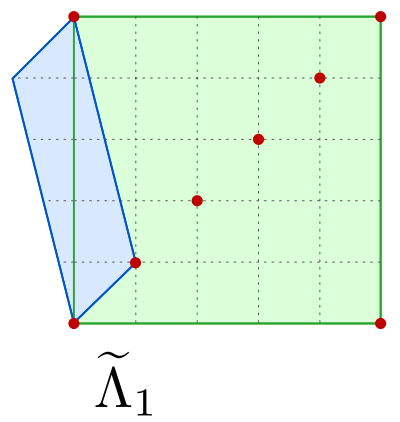

2) $G_{2} \simeq \mathbb{Z}_{8}$

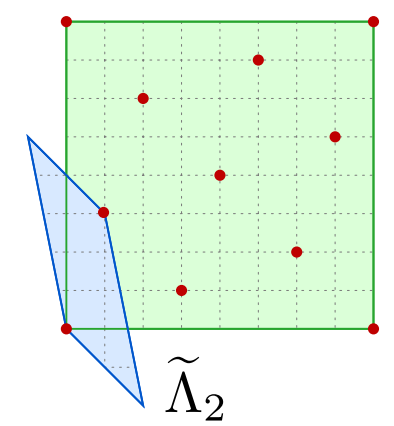

3) $G_{3} \simeq \mathbb{Z}_{12} \simeq \mathbb{Z}_{3} \times \mathbb{Z}_{4}$

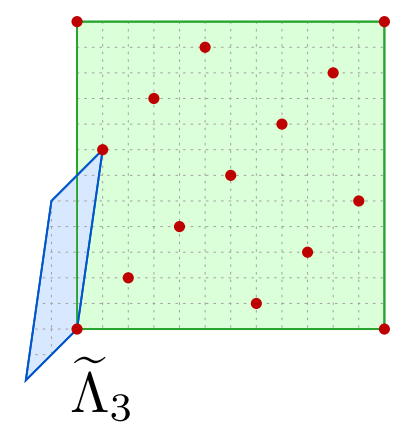

Figure 6. The unit cells $\widetilde{\Lambda}_{\ell}$ of each lattice $\widetilde{\Gamma}_{\ell}$ and their corresponding groups $G_{\ell} \simeq \widetilde{\Gamma}_{\ell} / \Gamma$. The small parallelograms represent the lattice generators (5.46) (where $\widetilde{\Lambda}_{1}$ has been shifted by a right $G L(2, \mathbb{Z})$ action in order to make it fit in the figure). The heavy red dots represent the members of each group $G_{\ell}$. The corners of the large squares are to be identified; they represent the lattice $\Gamma$ of the natural $2 \pi$ identifications of the $(\alpha, \beta)$ coordinates in $\mathbb{R}^{4}$.

We will first analyze the groups at these orbifold points. We find that the lattice generators $\widetilde{\Lambda}_{\ell}$, calculated from (4.47), are given by

$$
\widetilde{\Lambda}_{1}=\frac{1}{5}\left(\begin{array}{cc}
2 & -5 \\
-3 & 10
\end{array}\right), \quad \widetilde{\Lambda}_{2}=\frac{1}{8}\left(\begin{array}{cc}
2 & -1 \\
-2 & 5
\end{array}\right), \quad \widetilde{\Lambda}_{3}=\frac{1}{12}\left(\begin{array}{ll}
-2 & 7 \\
-2 & 1
\end{array}\right),
$$

and the corresponding groups are

$$
G_{1} \simeq \mathbb{Z}_{5}^{\text {diag }}, \quad G_{2} \simeq \mathbb{Z}_{8}, \quad G_{3} \simeq \mathbb{Z}_{12} \simeq \mathbb{Z}_{3} \times \mathbb{Z}_{4},
$$

where $G_{1}$ at point $\eta_{1}$ acts in the diagonal $\mathrm{U}(1)$ of $\mathrm{SO}(4)$, which one can check using (4.54). These lattice generators $\widetilde{\Lambda}_{\ell}$, and the groups given by $G_{\ell} \simeq \widetilde{\Gamma}_{\ell} / \Gamma$, are illustrated in figure 6 .

Next, we put the general expression for $m_{0}$ (B.22) into the bubble equations (5.33) and solve them for the $r_{i j}$, subject to the triangle constraint

$$
r_{12}+r_{23}=r_{13}
$$

At this point in the process it is quite possible to fail to find a solution. The $r_{i j}$ should be strictly positive (they do not enter the equations in a way that allows them to be treated as "directional"). The bubble equations are linear in $1 / r_{i j}$, and (5.48) is linear in $r_{i j}$, hence one is solving a system of quadratic equations. Thus it is possible to get negative or imaginary $r_{i j}$, and if this happens, one must adjust some of the dipole charges in (5.42) and try again. For the particular charges used here, we obtain two solution sets of real, positive $r_{i j}$, from which we select (via hindsight) the following:

$$
r_{12}=2.45827, \quad r_{23}=0.891937, \quad r_{13}=3.35021 .
$$

From this solution and the expressions (B.22) and (B.23), we then find

$$
m_{0}=1.96384, \quad \omega_{0}=-3.60037,
$$



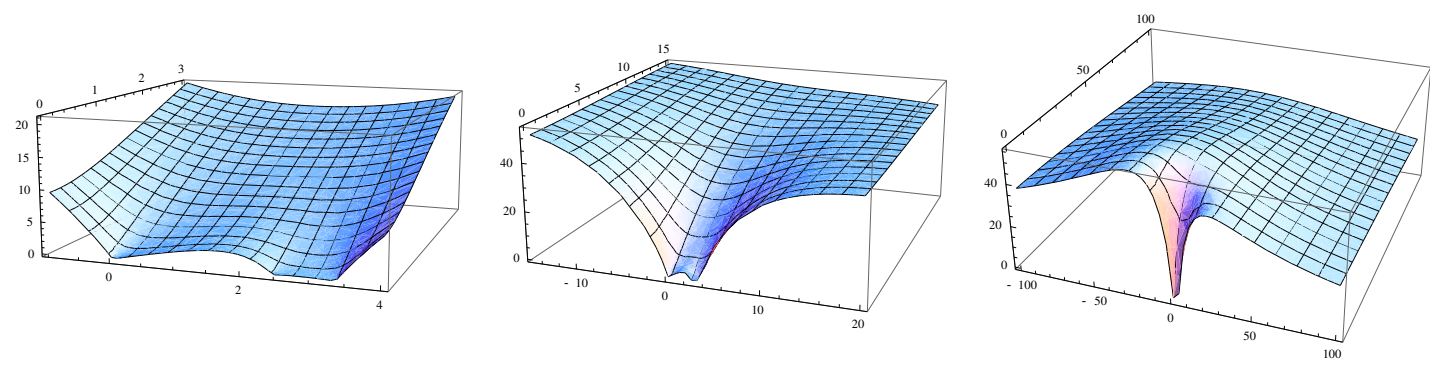

Figure 7. The function $\mathcal{Q} \equiv Z_{1} Z_{2} Z_{3} w-w^{2} \mu^{2}$ plotted near the source points at three different levels of magnification. $\mathcal{Q}$ is everywhere non-negative, and therefore the solution is free of CTC's.

which will then guarantee that there are no CTC's at infinity.

Finally, to show there are no CTC's anywhere, we plot

$$
\mathcal{Q} \equiv Z_{1} Z_{2} Z_{3} w-w^{2} \mu^{2}
$$

in figure 7. We see that it is positive near the centers as we expect, and appears to be positive everywhere, giving us a supergravity solution which is globally free of closed timelike curves. ${ }^{14}$

\section{Conclusions}

Using the floating brane ansatz of [20] we have obtained a new, infinite family of solutions to 5 -dimensional $\mathcal{N}=2$ ungauged supergravity coupled to two vector multiplets. To build the solutions, we start with a LeBrun metric for the 4-dimensional base. These metrics are Kähler and solve the Euclidean-Einstein-Maxwell equations, and are specified by two functions that solve the $\mathrm{SU}(\infty)$ Toda equation and its linearization. The full supergravity solution is then constructed by solving the "floating brane equations" on this base space. To these equations we obtain general, explicit solutions which generically represent a collection of concentric black rings stabilized by their angular momentum and electromagnetic charges. Under appropriate regularity conditions, the black rings are replaced by topological "bubbles", and the solutions are smooth and horizon-free. Imposing causality conditions, we obtain "bubble equations" which dictate the sizes of topological bubbles in terms of the cohomological fluxes trapped on them.

The 4-dimensional Kähler base space is interesting in its own right, and we spend some time analyzing its properties. Choosing a subclass of LeBrun metrics with $\mathrm{U}(1) \times \mathrm{U}(1)$ symmetry, we are able to solve the Toda equation and write down an explicit metric. Like the Gibbons-Hawking metrics, these metrics have an explicit U(1) fiber that pinches off at various points along the axis to create a series of homological 2-spheres. However, a new feature of the LeBrun metrics is that homological 2-spheres can also be formed by the other angular coordinate, and we obtain the specific boundary conditions that allow this to happen. We also find a new feature as we approach the Gibbons-Hawking points, or

\footnotetext{
${ }^{14}$ Naturally, it is not enough just to look at graphs. It is also helpful to plot $\mathcal{Q}-|\mathcal{Q}|$, which quickly reveals any place $\mathcal{Q}$ might go negative. This was checked in this example, and $\mathcal{Q} \geq 0$ everywhere.
} 
"geometric charges." In the GH metric, the U(1) near these points fibers over the $S^{2}$ in the base to give $S^{3} / \mathbb{Z}_{q}$, which makes the local metric an orbifold $\mathbb{R}^{4} / \mathbb{Z}_{q}$. In the LeBrun metric, however, one generically has $\mathbb{R}^{4} / G$ at these points, where $G \simeq \mathbb{Z}_{m} \times \mathbb{Z}_{n}$ acts on the two angular coordinates in $\mathbb{R}^{4} \simeq \mathbb{R}^{2} \times \mathbb{R}^{2}$. Finally, and perhaps most importantly, the explicit LeBrun metrics obtained have a Maxwell field which is non-trivially threaded through its various 2-cycles. This allows rich new phenomena in the full supergravity solution that were not present in previous work by the author and collaborators [22, 23].

Looking at the full supergravity solution, we see a striking similarity between these nonsupersymmetric solutions and the previous, well-known BPS solutions [7]. The regularity conditions take virtually the same form. By demanding the absence of CTC's, we also obtain "bubble equations" which have largely the same features as in the BPS solutions: a 2 -cycle is held open by the product of the three flavors of fluxes threading it. However, the non-BPS bubble equations at a given point involve not only the fluxes on cycles adjacent to that point, but also involve all the fluxes on the nonadjacent cycles (which is a radical departure from the BPS bubble equations). This indicates new physics that was not present in the BPS case, involving a richer variety of $E \times B$ type interactions.

It is known from previous work that these 5 -dimensional non-supersymmetric solutions on a Kähler base are actually trivial KK reductions of BPS solutions in the 6-dimensional IIB frame [27-29]. This explains some of the features we see, and yet makes others more mysterious. It seems clear that the 5-dimensional solutions are force-balanced by a kind of "supersymmetry without supersymmetry" [32], and in fact might be closer to BPS than the so-called "almost BPS" solutions [14-16]. For example, the bubble equations here and in the traditional $5 \mathrm{~d}$ BPS solutions are both linear in the inverse distances $1 / r_{i j}$, whereas the "almost BPS" bubble equations are cubic in the inverse distances. Still, there are important differences between these bubble equations and the 5d BPS bubble equations that must be explained if we are to think of these as "secretly BPS."

Having found the non-BPS bubble equations, we also find that imposing the asymptotics of the near-horizon BMPV metric [26] precludes the existence of any finitely-sized bubbled solutions. However, one can see the near-horizon BMPV-like metrics as the result of a limiting process of scaling solutions [17, 42, 43]. Alternatively, one can lift to the $6 \mathrm{~d}$ IIB frame where one can allow different asymptotic behavior in one of the warp factors, and in this case one can find an infinite family of smooth geometries, with finitely-sized bubbles held open by their cohomological fluxes, which are asymptotic to a momentum wave solution on $A d S_{3} \times S^{3}$.

It would be interesting to explore further the lift to the $6 \mathrm{~d}$ IIB frame, as was done with the LeBrun-Burns metrics in [23]. In 6 dimensions, one has the possibility of regular supertubes, and one might also get a better handle on why the bubble equations differ between here and the traditional setting (particularly in containing non-local interactions).

It would also be interesting to look for an asymptotically-flat completion of these solutions in 5 dimensions by relaxing the simplifying assumptions used in the floating brane ansatz [20]. This is certainly a non-trivial thing to do, as one will likely be forced to address the full Einstein equations. 
Finally, we also point out that while this work has focused on smooth solutions, one also has within the same solution set an infinite family of singular solutions, representing various collections of non-supersymmetric, yet force-balanced, spinning 3-charge black rings.

We have presented here a number of results and techniques which we hope yield insight into supergravity and black hole microstates. Recent progress in the ability to find supergravity solutions is very exciting and full of possibilities, and it is clear that there are many avenues waiting to be explored.

\section{Acknowledgments}

I would like to thank Nick Warner, Iosif Bena, Juan Maldacena, and Gary Horowitz for useful conversations. I am also grateful to IPhT, CEA-Saclay, the Simons Foundation, the organizers of the String Theory and Gravity workshops in Benasque, Spain, and the organizers of the "Fuzz or Fire?" workshop at KITP, Santa Barbara, for their hospitality and stimulating atmosphere where parts of this work were completed. This work is supported in part by the DOE grant DE-FG03-84ER-40168.

\section{A Groups at conical points from lattices in $\mathrm{SO}(4)$}

In this section we discuss how to compute the orbifold structure at the conical singularities of the LeBrun metrics. We stress that not every conical singularity is an orbifold singularity. For a point to be an orbifold singularity, the geometry must approach $\mathbb{R}^{4} / G$ for some finite group $G \subset \mathrm{SO}(4)$; however, for generic values of the parameters, one can also obtain more general conical singularities that cannot be locally modeled as a quotient space of $\mathbb{R}^{4}$. To illustrate the difference, consider two different 2-dimensional cone metrics:

$$
\mathrm{d} s_{A}^{2}=\mathrm{d} r^{2}+r^{2} \frac{\mathrm{d} \theta^{2}}{n^{2}}, \quad \mathrm{~d} s_{B}^{2}=\mathrm{d} r^{2}+r^{2} \frac{m^{2} \mathrm{~d} \theta^{2}}{n^{2}}, \quad \theta \sim \theta+2 \pi,
$$

for $m, n>0 \in \mathbb{Z}$ relatively prime. In the first metric $\mathrm{d} s_{A}^{2}$, a circuit around the tip of the cone subtends $2 \pi / n$ radians; hence an $n$-fold cover of this space will fill out the standard $\mathbb{R}^{2}$, and this is the quotient space $\mathbb{R}^{2} / \mathbb{Z}_{n}$. In the second metric $\mathrm{d} s_{B}^{2}$, however, a path enclosing the origin subtends $2 \pi m / n$ radians, and there is no $p$-fold cover of this space that gives us $\mathbb{R}^{2}$; hence it is not a quotient of $\mathbb{R}^{2}$, and not, strictly speaking, an orbifold. A similar phenomenon affects LeBrun metrics, except that there are two angular coordinates involved rather than one.

\section{A.1 Orbifold points and more general conical singularities}

Near each conical point in the LeBrun metric, one finds that the (local) metric approaches that of flat $\mathbb{R}^{4}$, but with the $\mathrm{U}(1) \times \mathrm{U}(1)$ coordinates identified on a lattice $\widetilde{\Gamma}$ different from the usual one $\Gamma$. One can define a group structure $G$, which is a finite subgroup of $\mathrm{U}(1) \times \mathrm{U}(1) \subset \mathrm{SO}(4)$, by comparing the two lattices $\Gamma, \widetilde{\Gamma}$. The conical point is an orbifold point precisely when $\Gamma \subseteq \widetilde{\Gamma}$ as a sublattice, and then the local geometry approaches $\mathbb{R}^{4} / G$. In this section we will compute $G$. 


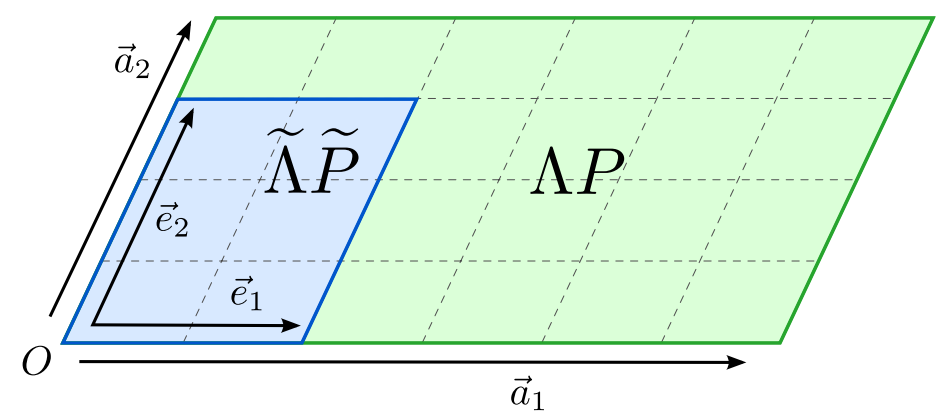

Figure 8. The lattice bases $\Lambda P$ and $\widetilde{\Lambda} \widetilde{P}$ are parallel. There exist rational numbers $r_{1}, r_{2}$ such that $\vec{a}_{1}=r_{1} \vec{e}_{1}$ and $\vec{a}_{2}=r_{2} \vec{e}_{2}$. In this case $r_{1}=3$ and $r_{2}=4 / 3$.

Let $\Gamma$ be the standard lattice on which to identify the $\mathrm{U}(1) \times \mathrm{U}(1)$ coordinates of $\mathbb{R}^{4}$. In the coordinates

$$
\mathrm{d} s^{2}\left(\mathbb{R}^{4}\right)=\mathrm{d} \rho^{2}+\rho^{2}\left(\mathrm{~d} \theta^{2}+\cos ^{2} \theta \mathrm{d} \alpha^{2}+\sin ^{2} \theta \mathrm{d} \beta^{2}\right),
$$

one has $(\alpha, \beta) \sim(\alpha+2 \pi, \beta) \sim(\alpha, \beta+2 \pi)$, and hence the basis $\Lambda$ of $\Gamma$ can be written

$$
\Lambda=2 \pi\left(\begin{array}{ll}
1 & 0 \\
0 & 1
\end{array}\right)
$$

where the columns are the two basis vectors. We note that $\Lambda$ is only defined up to right action by $G L(2, \mathbb{Z})$, because we are free to choose any two column vectors that generate the same lattice.

We should then compare this lattice $\Gamma$ to the lattice $\widetilde{\Gamma}$ of coordinate identifications obtained from the near-singularity limit of the LeBrun metric (after transforming it into the same $\mathbb{R}^{4}$ coordinates as above).

\section{A.1.1 Reduction to Smith normal form}

The lattices $\Gamma, \widetilde{\Gamma}$ have unit cells which are parallelograms of any dimensions and oriented in any directions. Let $\Lambda, \widetilde{\Lambda}$ be a choice of basis for each of $\Gamma, \widetilde{\Gamma}$. Since the lattices are rational to each other, we can always make a change of basis via right action by $P, \widetilde{P} \in G L(2, \mathbb{Z})$ such that the new bases $\Lambda P, \widetilde{\Lambda} \widetilde{P}$ are parallel, by which we mean

$$
\widetilde{\Lambda} \widetilde{P} R=\Lambda P, \quad \text { where } \quad R=\left(\begin{array}{cc}
r_{1} & 0 \\
0 & r_{2}
\end{array}\right),
$$

for some rational numbers $r_{1}, r_{2}>0$. This is shown in figure 8 .

The rational numbers $r_{1}, r_{2}$ give the factors by which each leg of $\Lambda P$ is larger than the same leg of $\widetilde{\Lambda} \widetilde{P}$. It is easy to see that each leg of $\widetilde{\Lambda} \widetilde{P}$ generates a cyclic group modulo the unit cell $\Lambda P$, and hence one has

$$
G \simeq \mathbb{Z}_{m} \times \mathbb{Z}_{n}, \quad \text { where } \quad m=\frac{r_{1}}{\operatorname{gcd}\left(1, r_{1}\right)}, \quad n=\frac{r_{2}}{\operatorname{gcd}\left(1, r_{2}\right)} .
$$


An orbifold point occurs precisely when $r_{1}, r_{2}$ are integers, in which case the lattice cell $\widetilde{\Lambda}$ "fits into" $\Lambda$ evenly. Then (A.5) can be written simply

$$
G \simeq \mathbb{Z}_{m} \times \mathbb{Z}_{n}, \quad \text { where } \quad m=r_{1}, \quad n=r_{2} .
$$

That is, at an orbifold point, the entries in the diagonal matrix $R$ give the orders of $\mathbb{Z}_{m}, \mathbb{Z}_{n}$.

What is left is to find $r_{1}, r_{2}$ in the first place. To do this, one takes (A.4) and isolates the diagonal matrix $R$ :

$$
R=\widetilde{P}^{-1} \widetilde{\Lambda}^{-1} \Lambda P .
$$

We do not need to know $P, \widetilde{P} \in G L(2, \mathbb{Z})$ explicitly; we merely need to describe an algorithm for diagonalizing $\widetilde{\Lambda}^{-1} \Lambda$ by independent actions of $G L(2, \mathbb{Z})$ from both the left and the right. This is precisely the algorithm for finding the Smith normal form of a matrix. Since we have available both left and right $G L(2, \mathbb{Z})$ actions, we may apply any sequence of elementary row or column operations which are invertible over $\mathbb{Z}$.

Hence to obtain $R$ we diagonalize $\widetilde{\Lambda}^{-1} \Lambda$ via the following process. At every step of the algorithm, we may

1. Swap any two rows or any two columns, or

2. Multiply any row, or any column, by -1 , or

3. Add an integer multiple of any row (column) to another row (column).

The objective is to reach a diagonal matrix (this is always possible). The full algorithm for the Smith normal form continues until the matrix is not only diagonal, but each entry along the diagonal divides the next, i.e. $r_{1} \mid r_{2}$ in this case. For our purposes, however, any diagonal matrix will do (and the result may not be unique).

In the case where the result is not unique, different possible results $R$ yield different ways of writing the same group $G$. For example, a given matrix might be diagonalized in two different ways to give $G \simeq \mathbb{Z}_{4} \times \mathbb{Z}_{6}$ or $G \simeq \mathbb{Z}_{2} \times \mathbb{Z}_{12}$, but these groups are isomorphic. The same matrix cannot also be diagonalized to give, e.g. $\mathbb{Z}_{3} \times \mathbb{Z}_{8}$ - the algorithm as constructed preserves the group structure. ${ }^{15}$

Once we have obtained $R$, we can then calculate the group $G$ via (A.5). We note that the order of $G$ is

$$
\# G=m n=\frac{r_{1}}{\operatorname{gcd}\left(1, r_{1}\right)} \times \frac{r_{2}}{\operatorname{gcd}\left(1, r_{2}\right)} \geq \frac{r_{1} r_{2}}{\operatorname{gcd}\left(1, r_{1} r_{2}\right)} .
$$

But $r_{1} r_{2}=\operatorname{det} R=\operatorname{det}\left(\widetilde{\Lambda}^{-1} \Lambda\right)$. Hence in terms of our lattice bases, we can put a lower bound on $\# G$ :

$$
\# G \geq \frac{\operatorname{det} \Lambda}{\operatorname{gcd}(\operatorname{det} \Lambda, \operatorname{det} \widetilde{\Lambda})},
$$

\footnotetext{
${ }^{15}$ Specifically, the reduction to Smith normal form of a square matrix $M$ preserves the sequence of invariant factors $r_{1}\left|r_{2}\right| \ldots \mid r_{n}$ such that $\operatorname{det} M=r_{1} r_{2} \ldots r_{n}$ and each $r_{i} \mid r_{i+1}$. It is precisely this sequence that distinguishes when the direct product of cyclic groups $\mathbb{Z}_{r_{1}} \times \mathbb{Z}_{r_{2}} \times \ldots \times \mathbb{Z}_{r_{n}}$ is isomorphic to another direct product of the same order.
} 
where we assume, without loss of generality, that $\operatorname{det} \Lambda, \operatorname{det} \widetilde{\Lambda}>0$ (which can always be arranged by the right action of $G L(2, \mathbb{Z})$ ). We note further that, at an orbifold point where $r_{1}, r_{2} \in \mathbb{Z}$, the inequality (A.9) is saturated, and then we can calculate the order of the group $G$ directly from the invariants $\operatorname{det} \Lambda, \operatorname{det} \widetilde{\Lambda}$.

\section{A.2 The conical points of LeBrun metrics}

In this section we will find the groups $G$ at the conical points of the LeBrun metric using the methods outlined in the previous section.

Near the conical points, the LeBrun metric approaches the form (4.41)

$$
\mathrm{d} s^{2}(L B)=\mathrm{d} \varrho^{2}+\frac{\varrho^{2}}{4}\left[\mathrm{~d} \theta^{2}+\frac{1}{\widetilde{q}_{\ell}^{2}}\left(\widetilde{K}(\theta) \mathrm{d} \tau^{2}-2 \widetilde{K Q}(\theta) \mathrm{d} \tau \mathrm{d} \phi+\widetilde{Q}(\theta) \mathrm{d} \phi^{2}\right)\right],
$$

and one must then compare it to a standard flat metric on $\mathbb{R}^{4}$,

$$
\mathrm{d} s^{2}\left(\mathbb{R}^{4}\right)=\mathrm{d} \varrho^{2}+\varrho^{2}\left(\mathrm{~d} \vartheta^{2}+\cos ^{2} \vartheta \mathrm{d} \alpha^{2}+\sin ^{2} \vartheta \mathrm{d} \beta^{2}\right),
$$

where $\theta=2 \vartheta$. From the LeBrun coordinates $(\tau, \phi)$, one can go to $(\alpha, \beta)$ via

$$
\begin{aligned}
& \alpha=\frac{1}{2 \widetilde{q}_{\ell}}\left(\left(k_{\ell}^{3}+\bar{K}_{\ell}^{3}-k_{0}^{3}\right) \tau-\left(q_{\ell}+\bar{Q}_{\ell}-q_{0}\right) \phi\right), \\
& \beta=\frac{1}{2 \widetilde{q}_{\ell}}\left(\left(k_{\ell}^{3}-\bar{K}_{\ell}^{3}+k_{0}^{3}\right) \tau-\left(q_{\ell}-\bar{Q}_{\ell}+q_{0}\right) \phi\right) .
\end{aligned}
$$

We need to define a "standard" lattice $\Gamma_{L B}$ on which the LeBrun coordinates $(\tau, \phi)$ should be identified in the first place. This is actually an arbitrary choice (it will merely affect how we interpret the various parameters $\left.q_{\ell}, k_{\ell}^{3}\right)$. But it is natural to borrow the standard "diamond lattice" from Gibbons-Hawking metrics:

$$
(\tau, \phi): \quad(0,0) \sim(4 \pi, 0) \sim(2 \pi, 2 \pi) \sim(2 \pi,-2 \pi) .
$$

By following the identifications (A.14) along the coordinate transformation (A.12), (A.13), we obtain the lattice $\widetilde{\Gamma}$ in the coordinates $(\alpha, \beta)$ given by the basis

$$
\widetilde{\Lambda}=2 \pi \cdot \frac{1}{2 \widetilde{q}_{\ell}}\left(\begin{array}{ll}
k_{\ell}^{3}+\hat{K}_{\ell}^{3}+q_{\ell}+\hat{Q}_{\ell} & k_{\ell}^{3}+\hat{K}_{\ell}^{3}-q_{\ell}-\hat{Q}_{\ell} \\
k_{\ell}^{3}-\hat{K}_{\ell}^{3}+q_{\ell}-\hat{Q}_{\ell} & k_{\ell}^{3}-\hat{K}_{\ell}^{3}-q_{\ell}+\hat{Q}_{\ell}
\end{array}\right),
$$

where for ease of legibility we have defined

$$
\hat{K}_{\ell}^{3} \equiv \bar{K}_{\ell}^{3}-k_{0}^{3}, \quad \hat{Q}_{\ell} \equiv \bar{Q}_{\ell}-q_{0} .
$$

The standard lattice $\Gamma$ in the coordinates $(\alpha, \beta)$ is given simply by the basis

$$
\Lambda=2 \pi\left(\begin{array}{ll}
1 & 0 \\
0 & 1
\end{array}\right),
$$

which makes the calculations easy, as $\widetilde{\Lambda}^{-1} \Lambda$ is just $2 \pi \widetilde{\Lambda}^{-1}$.

From (A.9), we see that the order of the group $G$ is at least $\left|\widetilde{q}_{\ell}\right|$ :

$$
\operatorname{det}\left(\widetilde{\Lambda}^{-1} \Lambda\right)=-\widetilde{q}_{\ell}, \quad \text { and hence } \# G \geq\left|\widetilde{q}_{\ell}\right|,
$$

And if $r_{1}, r_{2} \in \mathbb{Z}$, we have simply

$$
\# G=\left|\widetilde{q}_{\ell}\right| \text { at orbifold points. }
$$




\section{A.2.1 When is a conical point an orbifold point?}

As we have pointed out, an orbifold point occurs when $r_{1}, r_{2} \in \mathbb{Z}$, or alternatively, when $\widetilde{\Lambda}^{-1} \Lambda \in \operatorname{Mat}_{2}(\mathbb{Z})$, the set (not group) of $2 \times 2$ matrices with integer entries. This yields the condition

$$
\frac{1}{2}\left(\begin{array}{cc}
k_{\ell}^{3}-\hat{K}_{\ell}^{3}-q_{\ell}+\hat{Q}_{\ell} & -k_{\ell}^{3}-\hat{K}_{\ell}^{3}+q_{\ell}+\hat{Q}_{\ell} \\
-k_{\ell}^{3}+\hat{K}_{\ell}^{3}-q_{\ell}+\hat{Q}_{\ell} & k_{\ell}^{3}+\hat{K}_{\ell}^{3}+q_{\ell}+\hat{Q}_{\ell}
\end{array}\right) \in \operatorname{Mat}_{2}(\mathbb{Z}),
$$

where notably the $1 / \widetilde{q}_{\ell}$ in (A.15) has dropped out. Thus a LeBrun metric contains only orbifold points, and no generic conical points, when the sum of all the parameters is even:

$$
\left(k_{0}^{3}+\sum_{i=1}^{N} k_{i}^{3}+q_{0}+\sum_{i=1}^{N} q_{i}\right) \in 2 \mathbb{Z} .
$$

Conversely, none of the conical points have the quotient structure $\mathbb{R}^{4} / G$ if the sum of parameters is odd. We will assume this sum is even such that each conical point is an orbifold point with structure $\mathbb{R}^{4} / G$.

\section{A.2.2 When is the group $G$ trivial?}

The group $G$ is trivial whenever $\widetilde{\Gamma}, \Gamma$ are the same lattice. This happens whenever $\widetilde{\Lambda}^{-1} \Lambda \in$ $G L(2, \mathbb{Z})$. That is,

$$
\frac{1}{2}\left(\begin{array}{cc}
k_{\ell}^{3}-\hat{K}_{\ell}^{3}-q_{\ell}+\hat{Q}_{\ell} & -k_{\ell}^{3}-\hat{K}_{\ell}^{3}+q_{\ell}+\hat{Q}_{\ell} \\
-k_{\ell}^{3}+\hat{K}_{\ell}^{3}-q_{\ell}+\hat{Q}_{\ell} & k_{\ell}^{3}+\hat{K}_{\ell}^{3}+q_{\ell}+\hat{Q}_{\ell}
\end{array}\right) \in G L(2, \mathbb{Z}),
$$

The factor of $1 / 2$ imposes the parity condition (A.21). Furthermore, the determinant of this matrix is $\widetilde{q}_{\ell} \equiv q_{\ell} \hat{K}_{\ell}^{3}-k_{\ell}^{3} \hat{Q}_{\ell}$. Therefore for the metric to locally look like $\mathbb{R}^{4}$ with no conical singularity requires

$$
\widetilde{q}_{\ell}= \pm 1
$$

\section{A.2.3 When is the group $G$ like a Gibbons-Hawking orbifold group?}

A 1-center Gibbons-Hawking metric with "charge" $m$, written

$$
\mathrm{d} s^{2}(G H)=\frac{r}{m}(\mathrm{~d} \psi+m \cos \theta \mathrm{d} \chi)^{2}+\frac{m}{r}\left(\mathrm{~d} r^{2}+r^{2} \mathrm{~d} \theta^{2}+r^{2} \sin ^{2} \theta \mathrm{d} \chi^{2}\right),
$$

is a metric on the orbifold $\mathbb{R}^{4} / \mathbb{Z}_{m}$, where $\mathbb{Z}_{m}$ acts in the diagonal $\mathrm{U}(1)$ of the maximal torus $\mathrm{U}(1) \times \mathrm{U}(1) \in \mathrm{SO}(4)$. In $(\alpha, \beta)$ coordinates, this corresponds to the lattice $\Gamma_{G H}$ with basis

$$
\Lambda_{G H}=2 \pi\left(\begin{array}{ll}
1 & \frac{p}{m} \\
0 & \frac{p}{m}
\end{array}\right)
$$

where $p$ and $m$ are relativaly prime. The LeBrun metric then has a "diagonal" orbifold point whenever $\widetilde{\Lambda}^{-1} \Lambda_{G H} \in G L(2, \mathbb{Z})$, or equivalently, whenever $\Lambda_{G H}^{-1} \widetilde{\Lambda} \in G L(2, \mathbb{Z})$, since the determinant is \pm 1 in any case. This requires first that

$$
\operatorname{det}\left(\Lambda_{G H}^{-1} \widetilde{\Lambda}\right)=-\frac{m}{p \widetilde{q}_{\ell}}= \pm 1, \quad \text { or } \quad m= \pm p \widetilde{q}_{\ell} .
$$


But since $p$ and $m$ are relatively prime, we must have $p=1$ and $\widetilde{q}_{\ell}=m$. Next, writing out $\Lambda_{G H}^{-1} \widetilde{\Lambda}$ we have

$$
\frac{1}{2 \widetilde{q}_{\ell}}\left(\begin{array}{cc}
2\left(\hat{K}_{\ell}^{3}+\hat{Q}_{\ell}\right) & 2\left(\hat{K}_{\ell}^{3}-\hat{Q}_{\ell}\right) \\
\widetilde{q}_{\ell}\left(k_{\ell}^{3}-\hat{K}_{\ell}^{3}+q_{\ell}-\hat{Q}_{\ell}\right) & \widetilde{q}_{\ell}\left(k_{\ell}^{3}-\hat{K}_{\ell}^{3}-q_{\ell}+\hat{Q}_{\ell}\right)
\end{array}\right) \in G L(2, \mathbb{Z}) .
$$

So again, the sum of all the parameters must be even, and one gets a "diagonal" orbifold point wherever

$$
\frac{2\left(\bar{K}_{\ell}^{3}-k_{0}^{3}\right)}{\widetilde{q}_{\ell}} \in \mathbb{Z} \quad \text { and } \quad \frac{2\left(\bar{Q}_{\ell}-q_{0}\right)}{\widetilde{q}_{\ell}} \in \mathbb{Z} .
$$

One may also consider $\mathbb{Z}_{m}$ acting in the "anti-diagonal" $\mathrm{U}(1)$, which in $(\alpha, \beta)$ coordinates corresponds to the lattice $\Gamma_{\overline{G H}}$ with basis

$$
\Lambda_{\overline{G H}}=2 \pi\left(\begin{array}{cc}
1 & -\frac{1}{m} \\
0 & \frac{1}{m}
\end{array}\right)
$$

One can similarly show that these points occur for $\widetilde{q}_{\ell}=m$ and

$$
\frac{2 k_{\ell}^{3}}{\widetilde{q}_{\ell}} \in \mathbb{Z} \quad \text { and } \quad \frac{2 q_{\ell}}{\widetilde{q}_{\ell}} \in \mathbb{Z}
$$

\section{B Solutions to the Floating Brane system}

In this section we will solve the Floating Brane equations on the axisymmetric LeBrun base.

First, the $L_{1}, L_{2}$ equations (3.20) are simply the linearized Toda equation, which we have already solved to obtain $w$. We define "potentials" in the same way as in (4.7),

$$
L_{1}=\partial_{z} \hat{L}_{1}, \quad L_{2}=\partial_{z} \hat{L}_{2}
$$

such that $\hat{L}_{1}, \hat{L}_{2}$ solve the cylindrically-symmetric Laplace equation:

$$
\begin{aligned}
\hat{L}_{1} & =\ell_{1}^{0} \log \rho+\sum_{i} \ell_{1}^{i} G_{i}(\rho, \eta), \quad \hat{L}_{2}=\ell_{2}^{0} \log \rho+\sum_{i} \ell_{2}^{i} G_{i}(\rho, \eta), \\
G_{i}(\rho, \eta) & =\log \frac{\eta-\eta_{i}+\sqrt{\rho^{2}+\left(\eta-\eta_{i}\right)^{2}}}{\rho}
\end{aligned}
$$

where sums are understood to run from 1 to $N$. Then $L_{1}, L_{2}$ can be written

$$
\begin{aligned}
& L_{1}=\frac{1}{\rho\left(V_{\rho \eta}^{2}+V_{\eta \eta}^{2}\right)}\left(V_{\eta \eta} \hat{L}_{1, \rho}-V_{\rho \eta} \hat{L}_{1, \eta}\right), \\
& L_{2}=\frac{1}{\rho\left(V_{\rho \eta}^{2}+V_{\eta \eta}^{2}\right)}\left(V_{\eta \eta} \hat{L}_{2, \rho}-V_{\rho \eta} \hat{L}_{2, \eta}\right) .
\end{aligned}
$$

The $K^{1}, K^{2}, M$ equations (3.21), (3.22), (3.24) are all similar to each other. On the left-hand side is the cylindrically-symmetric Laplace operator on $\mathbb{R}^{3}$, and on the right-hand side is a product of two functions that solve the linearized Toda equation. Writing down 
the obvious homogeneous part, and then making an appropriate guess to match the source terms, the solutions are

$$
\begin{aligned}
& K^{1}=k_{0}^{1}+\sum_{i} \frac{k_{i}^{1}}{\Sigma_{i}}+\frac{1}{V_{\rho \eta}^{2}+V_{\eta \eta}^{2}}\left(V_{\eta \eta}\left(\hat{w}_{\eta} \hat{L}_{2, \eta}-\hat{w}_{\rho} \hat{L}_{2, \rho}\right)+V_{\rho \eta}\left(\hat{w}_{\eta} \hat{L}_{2, \rho}+\hat{w}_{\rho} \hat{L}_{2, \eta}\right)\right), \\
& K^{2}=k_{0}^{2}+\sum_{i} \frac{k_{i}^{2}}{\Sigma_{i}}+\frac{1}{V_{\rho \eta}^{2}+V_{\eta \eta}^{2}}\left(V_{\eta \eta}\left(\hat{w}_{\eta} \hat{L}_{1, \eta}-\hat{w}_{\rho} \hat{L}_{1, \rho}\right)+V_{\rho \eta}\left(\hat{w}_{\eta} \hat{L}_{1, \rho}+\hat{w}_{\rho} \hat{L}_{1, \eta}\right)\right), \\
& M=m_{0}+\sum_{i} \frac{m_{i}}{\Sigma_{i}}+\frac{1}{2} \frac{1}{V_{\rho \eta}^{2}+V_{\eta \eta}^{2}}\left(V_{\eta \eta}\left(\hat{L}_{1, \eta} \hat{L}_{2, \eta}-\hat{L}_{1, \rho} \hat{L}_{2, \rho}\right)+V_{\rho \eta}\left(\hat{L}_{1, \eta} \hat{L}_{2, \rho}+\hat{L}_{1, \rho} \hat{L}_{2, \eta}\right)\right),
\end{aligned}
$$

where $\Sigma_{i} \equiv \sqrt{\rho^{2}+\left(\eta-\eta_{i}\right)^{2}}$.

The $L_{3}$ equation offers no shortcuts. After a tedious exercise, one can show its solution is

$$
\begin{aligned}
L_{3}=\ell_{3}^{0} & -\ell_{3}^{z} \rho V_{\rho}+\sum_{i} \frac{1}{\Sigma_{i}}\left(k_{0}^{3} \ell_{3}^{i}+\ell_{1}^{0} k_{i}^{1}+\ell_{2}^{0} k_{i}^{2}+2 q_{0} m_{i}\right) \\
& +\sum_{\substack{i j \\
i \neq j}} \frac{1}{\eta_{i}-\eta_{j}} \frac{\Sigma_{i}}{\Sigma_{j}}\left(k_{i}^{3} \ell_{3}^{j}+\ell_{1}^{i} k_{j}^{1}+\ell_{2}^{i} k_{j}^{2}+2 q_{i} m_{j}\right) \\
& -\sum_{i} \frac{\eta-\eta_{i}}{\Sigma_{i}}\left(k_{i}^{3} \ell_{3}^{i}+\ell_{1}^{i} k_{i}^{1}+\ell_{2}^{i} k_{i}^{2}+2 q_{i} m_{i}\right) \\
& +\frac{\rho}{V_{\rho \eta}^{2}+V_{\eta \eta}^{2}}\left[V_{\rho \eta}\left(-\hat{w}_{\eta} \hat{L}_{1, \eta} \hat{L}_{2, \eta}+\hat{w}_{\rho} \hat{L}_{1, \rho} \hat{L}_{2, \eta}+\hat{w}_{\rho} \hat{L}_{1, \eta} \hat{L}_{2, \rho}+\hat{w}_{\eta} \hat{L}_{1, \rho} \hat{L}_{2, \rho}\right)\right. \\
& \left.\quad+V_{\eta \eta}\left(-\hat{w}_{\rho} \hat{L}_{1, \rho} \hat{L}_{2, \rho}+\hat{w}_{\rho} \hat{L}_{1, \eta} \hat{L}_{2, \eta}+\hat{w}_{\eta} \hat{L}_{1, \rho} \hat{L}_{2, \eta}+\hat{w}_{\eta} \hat{L}_{1, \eta} \hat{L}_{2, \rho}\right)\right],
\end{aligned}
$$

where the parameter $\ell_{3}^{z}$ multiplies $z=-\rho V_{\rho}$. It is important to note here that the pair $k_{i}^{3}, \ell_{3}^{j}$ behaves oppositely to the pairs $\ell_{1}^{i}, k_{j}^{1}$ and $\ell_{2}^{i}, k_{j}^{2}$.

Finally, one must solve the $\omega$ equation (3.26). If we write

$$
\omega=\omega_{(\phi)} \mathrm{d} \phi
$$

then (3.26) reduces to the two equations

$$
\begin{aligned}
r \partial_{r}\left(\omega_{(\phi)}\right)= & \frac{1}{2}\left(\rho^{2} L_{1} \partial_{z} K^{1}-K^{1} \partial_{z}\left(\rho^{2} L_{1}\right)\right)+\frac{1}{2}\left(\rho^{2} L_{2} \partial_{z} K^{2}-K^{2} \partial_{z}\left(\rho^{2} L_{2}\right)\right) \\
& +\frac{1}{4}\left(L_{3} \partial_{z}^{2}\left(\rho^{2}\right)-\partial_{z}\left(\rho^{2}\right) \partial_{z} L_{3}\right)+\rho^{2} w \partial_{z} M-M \partial_{z}\left(\rho^{2} w\right)-2 \rho^{2} w L_{1} L_{2}, \\
-\partial_{z}\left(\omega_{(\phi)}\right)= & \frac{1}{2}\left(L_{1} r \partial_{r} K^{1}-K^{1} r \partial_{r} L_{1}\right)+\frac{1}{2}\left(L_{2} r \partial_{r} K^{2}-K^{2} r \partial_{r} L_{2}\right) \\
& +\frac{1}{4}\left(L_{3} r \partial_{r} u_{z}-u_{z} r \partial_{r} L_{3}\right)+w r \partial_{r} M-M r \partial_{r} w .
\end{aligned}
$$

It is again a tedious exercise to show that these are solved by

$$
\omega_{(\phi)}=\omega_{0}+\frac{1}{\rho^{2}\left(V_{\rho \eta}^{2}+V_{\eta \eta}^{2}\right)}\left\{\frac{1}{2} \ell_{3}^{z}\left(\rho^{2} V_{\rho} V_{\rho \eta}-\eta \rho^{2}\left(V_{\rho \eta}^{2}+V_{\eta \eta}^{2}\right)\right)\right.
$$




$$
\begin{aligned}
& +\frac{1}{2}\left(k_{0}^{1} \ell_{1}^{0}+k_{0}^{2} \ell_{1}^{0}-\ell_{3}^{0}+2 m_{0} q_{0}\right)\left(k_{0}^{3}-\sum_{i} \frac{\eta-\eta_{i}}{\Sigma_{i}} k_{i}^{3}\right) \\
& -\frac{1}{2} k_{0}^{3} \sum_{i}\left(k_{0}^{1} \ell_{1}^{i}+k_{0}^{2} \ell_{2}^{i}+2 m_{0} q_{i}\right) \frac{\eta-\eta_{i}}{\Sigma_{i}} \\
& +\frac{1}{2} \sum_{i j} k_{i}^{3}\left(k_{0}^{1} \ell_{1}^{j}+k_{0}^{2} \ell_{2}^{j}+2 m_{0} q_{j}\right) \frac{\rho^{2}+\left(\eta-\eta_{i}\right)\left(\eta-\eta_{j}\right)}{\Sigma_{i} \Sigma_{j}} \\
& +\frac{1}{2} k_{0}^{3} \sum_{\substack{i j \\
i \neq j}}\left(k_{i}^{1} \ell_{1}^{j}+k_{i}^{2} \ell_{2}^{j}-\ell_{3}^{i} k_{j}^{3}+2 m_{i} q_{j}\right) \frac{1}{\eta_{i}-\eta_{j}} \frac{\rho^{2}+\left(\eta-\eta_{i}\right)\left(\eta-\eta_{j}\right)}{\Sigma_{i} \Sigma_{j}} \\
& -\frac{1}{2} \sum_{\substack{i j k \\
i \neq j}} k_{k}^{3}\left(k_{i}^{1} \ell_{1}^{j}+k_{i}^{2} \ell_{2}^{j}+2 m_{i} q_{j}\right) \frac{1}{\eta_{i}-\eta_{j}} \frac{1}{\Sigma_{i} \Sigma_{j} \Sigma_{k}} \times \\
& +\frac{1}{2} \sum_{i k} k_{k}^{3}\left(k_{i}^{1} \ell_{1}^{i}+k_{i}^{2} \ell_{2}^{i}+2 m_{i} q_{i}\right) \frac{\rho^{2}}{\Sigma_{i}^{2} \Sigma_{k}} \\
& +\frac{1}{2} \sum_{\substack{i j k \\
i \neq k}} k_{i}^{3} k_{j}^{3} \ell_{3}^{k} \frac{\eta_{i}-\eta_{j}}{\eta_{i}-\eta_{k}} \frac{\rho^{2}}{\Sigma_{i} \Sigma_{j} \Sigma_{k}}-\frac{1}{2} \sum_{i j} k_{i}^{3} k_{j}^{3} \ell_{3}^{i} \frac{\rho^{2}}{\Sigma_{i}^{2} \Sigma_{j}}+\frac{1}{2} \sum_{i}\left(k_{i}^{3}\right)^{2} \ell_{3}^{i} \frac{\rho^{2}}{\Sigma_{i}^{3}} \\
& +\frac{1}{2} \sum_{\substack{i j k \\
i \neq k}} k_{i}^{3} k_{j}^{3} \ell_{3}^{k} \frac{1}{\eta_{i}-\eta_{k}} \frac{\left(\eta-\eta_{k}\right)\left(\rho^{2}+\left(\eta-\eta_{i}\right)\left(\eta-\eta_{j}\right)\right)}{\Sigma_{i} \Sigma_{j} \Sigma_{k}} \\
& \left.+\sum_{i j k} q_{i} \ell_{1}^{j} \ell_{2}^{j} \frac{\rho^{2}}{\Sigma_{i} \Sigma_{j} \Sigma_{k}}\right\}, \\
&
\end{aligned}
$$

where again, all sums are assumed to run over $i, j, k \in\{1 \ldots N\}$.

We now have the complete data for constructing supergravity solutions. The solution is characterized by $N$ number of points $\eta_{i}$ along the axis in the base space, and by the $8 N+10$ parameters $\left\{q_{0}, k_{0}^{1}, k_{0}^{2}, k_{0}^{3}, \ell_{1}^{0}, \ell_{2}^{0}, \ell_{3}^{0}, m_{0}, \omega_{0}, \ell_{3}^{z}, q_{i}, k_{i}^{1}, k_{i}^{2}, k_{i}^{3}, \ell_{1}^{i}, \ell_{2}^{i}, \ell_{3}^{i}, m_{i}\right\}$, which in general are constrained by the requirement for the absence of CTC's and DiracMisner strings. Finally, to complete the supergravity solution, one puts the functions $w, K^{1}, K^{2}, K^{3}, L_{1}, L_{2}, L_{3}, M$ into the ansätze of sections 2 and 3.3 .

\section{B.1 Asymptotic expansions of the metric functions}

In this section are the detailed asymptotic expansions of the metric functions in terms of the above solutions.

First, the parameters $k_{0}^{1}, k_{0}^{2}, \ell_{3}^{z}$ lead to terms that blow up at infinity, so we set

$$
k_{0}^{1}=0, \quad k_{0}^{2}=0, \quad \ell_{3}^{z}=0 .
$$

To look near infinity it is helpful to define the coordinates $R, \theta$ via

$$
\rho=R \sin \theta, \quad \eta=R \cos \theta .
$$


Then the warp factors $Z_{1}, Z_{2}$ go as $1 / R$ :

$$
Z_{1} \sim\left(\frac{K_{\star}^{2} K_{\star}^{3}+Q_{\star} L_{1}^{\star}}{q_{0} K_{\star}^{3}-k_{0}^{3} Q_{\star}}\right) \frac{1}{R}, \quad Z_{2} \sim\left(\frac{K_{\star}^{1} K_{\star}^{3}+Q_{\star} L_{2}^{\star}}{q_{0} K_{\star}^{3}-k_{0}^{3} Q_{\star}}\right) \frac{1}{R}
$$

where we define the quantities

$$
\begin{aligned}
K_{\star}^{1} \equiv \sum_{i=1}^{N} k_{i}^{1}, & K_{\star}^{2} \equiv \sum_{i=1}^{N} k_{i}^{2}, & K_{\star}^{3} \equiv \sum_{i=1}^{N} k_{i}^{3}, & Q_{\star} \equiv \sum_{i=1}^{N} q_{i}, \\
L_{1}^{\star} \equiv \sum_{i=1}^{N} \ell_{1}^{i}, & L_{2}^{\star} \equiv \sum_{i=1}^{N} \ell_{2}^{i}, & L_{3}^{\star} \equiv \sum_{i=1}^{N} \ell_{3}^{i}, & M_{\star} \equiv \sum_{i=1}^{N} m_{i} .
\end{aligned}
$$

At leading order, the remaining metric functions $Z_{3}, \mu, \omega_{(\phi)}$ go as constants:

$$
\begin{aligned}
Z_{3} \sim \ell_{3}^{0}-\sum_{\substack{i j \\
i \neq j}} \frac{k_{i}^{1} \ell_{1}^{j}+k_{i}^{2} \ell_{2}^{j}-k_{i}^{3} \ell_{3}^{j}+2 m_{i} q_{j}}{\eta_{i}-\eta_{j}}, \\
\mu \sim m_{0}-\frac{1}{2} \frac{K_{\star}^{3}}{q_{0} K_{\star}^{3}-k_{0}^{3} Q_{\star}}\left(\ell_{3}^{0}-\sum_{\substack{i j \\
i \neq j}} \frac{k_{i}^{1} \ell_{1}^{j}+k_{i}^{2} \ell_{2}^{j}-k_{i}^{3} \ell_{3}^{j}+2 m_{i} q_{j}}{\eta_{i}-\eta_{j}}\right), \\
\omega_{(\phi)} \sim \omega_{0}+\frac{1}{2} \frac{Q_{\star}}{q_{0} K_{\star}^{3}-k_{0}^{3} Q_{\star}}\left(\ell_{3}^{0}-\sum_{\substack{i j \\
i \neq j}} \frac{k_{i}^{1} \ell_{1}^{j}+k_{i}^{2} \ell_{2}^{j}-k_{i}^{3} \ell_{3}^{j}+2 m_{i} q_{j}}{\eta_{i}-\eta_{j}}\right) \\
+\left(\frac{k_{0}^{3} q_{0}+K_{\star}^{3} Q_{\star}-\left(q_{0} K_{\star}^{3}+k_{0}^{3} Q_{\star}\right) \cos \theta}{\left(k_{0}^{3}\right)^{2}+\left(K_{\star}^{3}\right)^{2}-2 k_{0}^{3} K_{\star}^{3} \cos \theta}\right) \times \\
\quad \times\left[m_{0}-\frac{1}{2} \frac{K_{\star}^{3}}{q_{0} K_{\star}^{3}-k_{0}^{3} Q_{\star}}\left(\ell_{3}^{0}-\sum_{i j} \frac{k_{i}^{1} \ell_{1}^{j}+k_{i}^{2} \ell_{2}^{j}-k_{i}^{3} \ell_{3}^{j}+2 m_{i} q_{j}}{\eta_{i}-\eta_{j}}\right)\right] .
\end{aligned}
$$

However, we must have $\mu \rightarrow 0, \omega_{(\phi)} \rightarrow 0$ asymptotically in order to avoid CTC's at infinity. Therefore we must set

$$
\begin{aligned}
& m_{0}=\frac{1}{2} \frac{K_{\star}^{3}}{q_{0} K_{\star}^{3}-k_{0}^{3} Q_{\star}}\left(\ell_{3}^{0}-\sum_{\substack{i j \\
i \neq j}} \frac{k_{i}^{1} \ell_{1}^{j}+k_{i}^{2} \ell_{2}^{j}-k_{i}^{3} \ell_{3}^{j}+2 m_{i} q_{j}}{\eta_{i}-\eta_{j}}\right), \\
& \omega_{0}=-\frac{1}{2} \frac{Q_{\star}}{q_{0} K_{\star}^{3}-k_{0}^{3} Q_{\star}}\left(\ell_{3}^{0}-\sum_{\substack{i j \\
i \neq j}} \frac{k_{i}^{1} \ell_{1}^{j}+k_{i}^{2} \ell_{2}^{j}-k_{i}^{3} \ell_{3}^{j}+2 m_{i} q_{j}}{\eta_{i}-\eta_{j}}\right) .
\end{aligned}
$$


Then in fact the asymptotic expansions of $\mu, \omega_{(\phi)}$ must be carried to the next order, giving

$$
\begin{gathered}
\mu \sim \frac{1}{R}\left\{\frac { 1 } { ( q _ { 0 } K _ { \star } ^ { 3 } - k _ { 0 } ^ { 3 } Q _ { \star } ) ^ { 2 } } \left[-K_{\star}^{3}\left(\left(k_{0}^{3}\right)^{2}+\left(K_{\star}^{3}\right)^{2}-2 k_{0}^{3} K_{\star}^{3} \cos \theta\right) K_{\star}^{1} K_{\star}^{2}\right.\right. \\
-K_{\star}^{3}\left(k_{0}^{3} q_{0}+K_{\star}^{3} Q_{\star}-2 k_{0}^{3} Q_{\star} \cos \theta\right)\left(K_{\star}^{1} L_{1}^{\star}+K_{\star}^{2} L_{2}^{\star}\right) \\
\left.-Q_{\star}\left(k_{0}^{3} q_{0}+K_{\star}^{3} Q_{\star}-\left(q_{0} K_{\star}^{3}+k_{0}^{3} Q_{\star}\right) \cos \theta\right) L_{\star}^{1} L_{\star}^{2}\right] \\
+\frac{1}{2} \frac{1}{q_{0} K_{\star}^{3}-k_{0}^{3} Q_{\star}}\left[\left(k_{0}^{3}+K_{\star}^{3} \cos \theta\right)\left(K_{\star}^{1} L_{1}^{\star}+K_{\star}^{2} L_{2}^{\star}\right)\right. \\
\left.\left.+\left(K_{\star}^{3} \cos \theta-k_{0}^{3}\right)\left(K_{\star}^{3} L_{3}^{\star}+2 Q_{\star} M_{\star}\right)\right]\right\},
\end{gathered}
$$

and

$$
\omega_{(\phi)} \sim \frac{1}{2 R} \frac{K_{\star}^{3} \sin ^{2} \theta}{\left(k_{0}^{3}\right)^{2}+\left(K_{\star}^{3}\right)^{2}-2 k_{0}^{3} K_{\star}^{3} \cos \theta}\left(K_{\star}^{1} L_{1}^{\star}+K_{\star}^{2} L_{2}^{\star}+K_{\star}^{3} L_{3}^{\star}+2 Q_{\star} M_{\star}\right) .
$$

Open Access. This article is distributed under the terms of the Creative Commons Attribution License (CC-BY 4.0), which permits any use, distribution and reproduction in any medium, provided the original author(s) and source are credited.

\section{References}

[1] J.D. Bekenstein, Black holes and entropy, Phys. Rev. D 7 (1973) 2333 [INSPIRE].

[2] S.W. Hawking, Black hole explosions, Nature 248 (1974) 30 [INSPIRE].

[3] S.D. Mathur, The fuzzball proposal for black holes: an elementary review, Fortsch. Phys. 53 (2005) 793 [hep-th/0502050] [INSPIRE].

[4] I. Bena and N.P. Warner, One ring to rule them all... And in the darkness bind them?, Adv. Theor. Math. Phys. 9 (2005) 667 [hep-th/0408106] [INSPIRE].

[5] I. Bena and N.P. Warner, Bubbling supertubes and foaming black holes, Phys. Rev. D 74 (2006) 066001 [hep-th/0505166] [INSPIRE].

[6] P. Berglund, E.G. Gimon and T.S. Levi, Supergravity microstates for BPS black holes and black rings, JHEP 06 (2006) 007 [hep-th/0505167] [INSPIRE].

[7] I. Bena and N.P. Warner, Black holes, black rings and their microstates, Lect. Notes Phys. 755 (2008) 1 [hep-th/0701216] [INSPIRE].

[8] G.W. Gibbons and N.P. Warner, Global structure of five-dimensional fuzzballs, Class. Quant. Grav. 31 (2014) 025016 [arXiv: 1305.0957] [InSPIRE].

[9] G.W. Gibbons and S.W. Hawking, Gravitational multi-instantons, Phys. Lett. B 78 (1978) 430 [INSPIRE].

[10] V. Jejjala, O. Madden, S.F. Ross and G. Titchener, Non-supersymmetric smooth geometries and D1-D5-P bound states, Phys. Rev. D 71 (2005) 124030 [hep-th/0504181] [INSPIRE].

[11] E.G. Gimon, T.S. Levi and S.F. Ross, Geometry of non-supersymmetric three-charge bound states, JHEP 08 (2007) 055 [arXiv:0705.1238] [INSPIRE]. 
[12] S. Giusto, S.F. Ross and A. Saxena, Non-supersymmetric microstates of the D1-D5-KK system, JHEP 12 (2007) 065 [arXiv:0708.3845] [INSPIRE].

[13] I. Bena, S. Giusto, C. Ruef and N.P. Warner, A (running) bolt for new reasons, JHEP 11 (2009) 089 [arXiv: 0909.2559] [INSPIRE].

[14] K. Goldstein and S. Katmadas, Almost BPS black holes, JHEP 05 (2009) 058 [arXiv: 0812.4183] [INSPIRE].

[15] I. Bena, G. Dall'Agata, S. Giusto, C. Ruef and N.P. Warner, Non-BPS black rings and black holes in Taub-NUT, JHEP 06 (2009) 015 [arXiv:0902.4526] [INSPIRE].

[16] I. Bena, S. Giusto, C. Ruef and N.P. Warner, Multi-center non-BPS black holes: the solution, JHEP 11 (2009) 032 [arXiv:0908.2121] [INSPIRE].

[17] O. Vasilakis and N.P. Warner, Mind the gap: supersymmetry breaking in scaling, microstate geometries, JHEP 10 (2011) 006 [arXiv:1104.2641] [INSPIRE].

[18] I. Bena, A. Puhm, O. Vasilakis and N.P. Warner, Almost BPS but still not renormalized, JHEP 09 (2013) 062 [arXiv:1303.0841] [INSPIRE].

[19] B.D. Chowdhury, D.R. Mayerson and B. Vercnocke, Phases of non-extremal multi-centered bound states, JHEP 12 (2013) 054 [arXiv:1307.5846] [INSPIRE].

[20] I. Bena, S. Giusto, C. Ruef and N.P. Warner, Supergravity solutions from floating branes, JHEP 03 (2010) 047 [arXiv:0910.1860] [INSPIRE].

[21] N. Bobev and C. Ruef, The nuts and bolts of Einstein-Maxwell solutions, JHEP 01 (2010) 124 [arXiv:0912.0010] [INSPIRE].

[22] N. Bobev, B. Niehoff and N.P. Warner, Hair in the back of a throat: non-supersymmetric multi-center solutions from Kähler manifolds, JHEP 10 (2011) 149 [arXiv:1103.0520] [INSPIRE].

[23] N. Bobev, B.E. Niehoff and N.P. Warner, New supersymmetric bubbles on $A d S_{3} \times S^{3}$, JHEP 10 (2012) 013 [arXiv: 1204.1972] [INSPIRE].

[24] C. LeBrun, Explicit self-dual metrics on $\mathbf{C P}_{2} \# \cdots \# \mathbf{C P}_{2}$, J. Diff. Geom. 34 (1991) 223.

[25] C. LeBrun, The Einstein-Maxwell equations, extremal Kähler metrics and Seiberg-Witten theory, arXiv:0803.3734 [INSPIRE].

[26] J.C. Breckenridge, R.C. Myers, A.W. Peet and C. Vafa, D-branes and spinning black holes, Phys. Lett. B 391 (1997) 93 [hep-th/9602065] [INSPIRE].

[27] J.B. Gutowski, D. Martelli and H.S. Reall, All supersymmetric solutions of minimal supergravity in six-dimensions, Class. Quant. Grav. 20 (2003) 5049 [hep-th/0306235] [INSPIRE].

[28] M. Cariglia and O.A.P. Mac Conamhna, The general form of supersymmetric solutions of $N=(1,0) \mathrm{U}(1)$ and $\mathrm{SU}(2)$ gauged supergravities in six-dimensions, Class. Quant. Grav. 21 (2004) 3171 [hep-th/0402055] [INSPIRE].

[29] I. Bena, S. Giusto, M. Shigemori and N.P. Warner, Supersymmetric solutions in six dimensions: a linear structure, JHEP 03 (2012) 084 [arXiv:1110.2781] [INSPIRE].

[30] J. Scherk and J.H. Schwarz, How to get masses from extra dimensions, Nucl. Phys. B 153 (1979) 61 [INSPIRE]. 
[31] J. Scherk and J.H. Schwarz, Spontaneous breaking of supersymmetry through dimensional reduction, Phys. Lett. B 82 (1979) 60 [InSPIRE].

[32] M.J. Duff, H. Lü and C.N. Pope, Supersymmetry without supersymmetry, Phys. Lett. B 409 (1997) 136 [hep-th/9704186] [INSPIRE].

[33] D. Gaiotto and J. Maldacena, The gravity duals of $N=2$ superconformal field theories, JHEP 10 (2012) 189 [arXiv:0904.4466] [INSPIRE].

[34] O. Aharony, L. Berdichevsky and M. Berkooz, $4 d N=2$ superconformal linear quivers with type IIA duals, JHEP 08 (2012) 131 [arXiv:1206.5916] [INSPIRE].

[35] R.A. Reid-Edwards and B. Stefański Jr., On type IIA geometries dual to $N=2$ SCFTs, Nucl. Phys. B 849 (2011) 549 [arXiv:1011.0216] [InSPIRE].

[36] O.P. Santillan and A.G. Zorin, Geometric origin of a linearization of the $\mathrm{SU}(\infty)$ Toda equation, Czech. J. Phys. 54 (2004) 1381.

[37] R.S. Ward, Einstein-Weyl spaces and SU( $\infty$ ) Toda fields, Class. Quant. Grav. 7 (1990) L95 [INSPIRE].

[38] M. Abreu and R. Sena-Dias, Scalar-flat Kähler metrics on non-compact symplectic toric 4-manifolds, arXiv:0910.5466.

[39] P.S. Aspinwall, Resolution of orbifold singularities in string theory, hep-th/9403123 [INSPIRE].

[40] G.W. Gibbons and C.N. Pope, The positive action conjecture and asymptotically euclidean metrics in quantum gravity, Commun. Math. Phys. 66 (1979) 267 [InSPIRE].

[41] S. Giusto, S.D. Mathur and A. Saxena, Dual geometries for a set of 3-charge microstates, Nucl. Phys. B 701 (2004) 357 [hep-th/0405017] [INSPIRE].

[42] I. Bena, C.-W. Wang and N.P. Warner, Plumbing the abyss: black ring microstates, JHEP 07 (2008) 019 [arXiv: 0706.3786] [INSPIRE].

[43] I. Bena, C.-W. Wang and N.P. Warner, Mergers and typical black hole microstates, JHEP 11 (2006) 042 [hep-th/0608217] [INSPIRE]. 